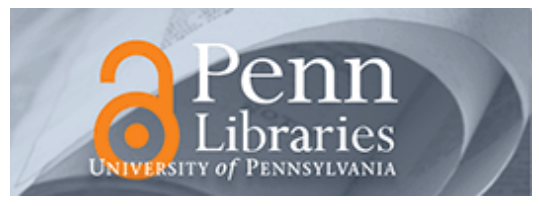

University of Pennsylvania

ScholarlyCommons

Finance Papers

Wharton Faculty Research

1998

\title{
Optimal Consumption Choices for a 'Large' Investor
}

Domenico Cuoco

University of Pennsylvania

Jakša Cvitanić

Follow this and additional works at: https://repository.upenn.edu/fnce_papers

Part of the Econometrics Commons, and the Finance and Financial Management Commons

\section{Recommended Citation}

Cuoco, D., \& Cvitanić, J. (1998). Optimal Consumption Choices for a 'Large' Investor. Journal of Economic Dynamics and Control, 22 (3), 401-436. http://dx.doi.org/10.1016/S0165-1889(97)00065-1

This paper is posted at ScholarlyCommons. https://repository.upenn.edu/fnce_papers/394

For more information, please contact repository@pobox.upenn.edu. 


\title{
Optimal Consumption Choices for a 'Large' Investor
}

\author{
Abstract \\ This paper examines the optimal consumption and investment problem for a 'large' investor, whose \\ portfolio choices affect the instantaneous expected returns on the traded assets. Alternatively, our \\ analysis can be interpreted in terms of an optimal growth problem with nonlinear technologies. Existence \\ of optimal policies is established using martingale and duality techniques under general assumptions on \\ the securities' price process and the investor's preferences. As an illustration of our characterization \\ result, explicit solutions are provided for specific examples involving an agent with logarithmic utilities \\ and a generalized two-factor version of the CCAPM is derived. The analogy of the consumption problem \\ examined in this paper to the consumption problem with constraints on the portfolio choices is \\ emphasized. \\ Disciplines \\ Econometrics | Finance and Financial Management
}




\title{
OPTIMAL CONSUMPTION CHOICES
}

FOR A "LARGE" INVESTOR

\author{
by \\ Domenico Cuoco \\ Jaksa Critanic
}

4-96

RODNEY L. WHITE CENTER FOR FINANCIAL RESEARCH The Wharton School University of Pennsylvania 3254 Steinberg Hall-Dietrich Hall

Philadelphia, PA 19104-6367

(215) 898-7616

The authors take full responsibility for the contents of this paper.

Copyright ${ }^{\circ} 1996$ by Domenico Cuoco and Jaksa Cvitanic 


\title{
ГOptimal Consumption Choices for a "Large" Investor"]
}

\author{
Domenico Cuoco \\ The Wharton School \\ University of Pennsylvania \\ Philadelphia, PA 19104 \\ cuoco@wharton.upenn.edu
}

\author{
Jakša Cvitanić \\ Department of Statistics \\ Columbia University \\ New York, NY 10027 \\ cj@stat.columbia.edu
}

Last revised: April 1996

\begin{abstract}
This paper examines the optimal consumption and investment problem for a "large" investor, whose portfolio choices affect the instantaneous expected returns on the traded assets. Alternatively, our analysis can be interpreted in terms of an optimal growth problem with nonlinear technologies. Existence of optimal policies is established using martingale and duality techniques under general assumptions on the securities' price process and the investor's preferences. As an illustration of our characterization result, explicit solutions are provided for specific examples involving an agent with logarithmic utilities, and a generalized two-factor version of the CCAPM is derived. The analogy of the consumption problem examined in this paper to the consumption problem with constraints on the portfolio choices is emphasized.
\end{abstract}

*We are grateful to Suleyman Basak and to participants in the finance workshop at the University of British Columbia, the economic theory workshop at Cornell University and the Micro Lunch Seminar at the .Wharton School for their comments.

Pino/He/136/P4/no $4-96$ 


\section{Introduction}

This paper examines the optimal consumption and investment problem for a "large" investor, whose portfolio choices affect the drift of the securities' price process. The impact of the investor's position on prices is specified exogenously and may arise because of size only or because other agents in the market believe that the "large" trader has superior information. Existence and characterization results for the optimal choices are obtained using martingale and duality techniques similar to those employed by He and Pearson (1991), Karatzas, Lehoczky, Shreve and Xu (1991), Xu and Shreve (1992a), Cvitanić and Karatzas (1992), He and Pagès (1993), among others, to analyze the consumption/investment problem in markets with constraints. In fact, the present framework with policy-dependent prices includes (at least formally) the case of portfolio constraints as a special case.

The correspondence of the optimal consumption problem with portfolio constraints to one with policy-dependent price drifts was first pointed out by El Karoui, Peng and Quenez (1995). They used the theory of backward stochastic differential equations, independently developed by Pardoux and Peng (1990) and Duffie and Epstein (1992), to analyze the problem of a "large" investor trying to maximize a recursive utility or to hedge a contingent claim. In particular, they provided a martingale characterization of optimal consumption plans and of the minimal cost to hedge a contingent claim, but did not prove the existence of an optimal consumption policy. Cvitanić and Ma (1994) pointed out that if the payoff of the contingent claim is not exogenously fixed, but is allowed to depend on the future price of some traded asset, then the hedging problem translates into finding a solution to a forwardbackward stochastic differential equation. For the Markovian case, the four-step scheme of $\mathrm{Ma}$, Protter and Yong (1994) was used to obtain a partial differential equation identifying the minimal hedging cost. Cvitanic (1995) surveys this literature and briefly discusses the difficulty (nonconvexity) that arises when trying to apply the duality approach of Cvitanić and Karatzas (1992) to establish the existence of an optimal consumption policy for a "large" investor. Needles to say, the existence of optimal policies is an important concern if a general dependence of price drifts on the investor portfolio choices is to be allowed, as arbitrage opportunities, or "market manipulation trading strategies", might arise. Jarrow $(1992,1994)$ has examined this issue in a discrete-time infinite-horizon economy with policydependent prices.

In this paper, we use martingale and duality techniques to provide sufficient conditions for the existence of an optimal consumption plan for a "large" investor and to characterize the optimal plan. The martingale duality approach maps the primal consumption/investment problem into a dual minimization problem that solves for the individual's shadow state-prices (intertemporal marginal rates of substitution). To cope with nonconvexity, we depart from the approach in the above-mentioned papers dealing with portfolio constraints, and establish the existence of optimal consumption/investment policies by formulating the dual problem directly over the space of shadow state-prices, rather than over 
the set of Lagrangian multipliers representing them. We illustrate our characterization of optimal consumption policies by providing closed-form solutions for specific examples involving an investor with logarithmic preferences. Moreover, we derive a generalized twofactor version of the CCAPM of Breeden (1979) valid in the presence of "large" investors.

Basak (1995) has recently examined the equilibrium implications of non-price-taking behavior. He allows the non-price-taking agent to directly choose the shadow price for the consumption good at any future date and state, subject only to a market-clearing condition. However, conditions guaranteeing the existence of optimal policies (or of an equilibrium) are not provided. Moreover, because of the way the agent is allowed to affect prices, the derived optimal policy is time-inconsistent. In this paper, the impact of the investor's choices on the assets' prices is postulated exogenously rather than derived from equilibrium considerations. On the other hand, we show that an optimal policy satisfying our characterization will indeed exist under fairly general conditions on the agent's preferences. Moreover, timeconsistency is preserved in our setting.

An alternative straightforward application of our results is to the theory of optimal growth in economies with nonlinear production technologies.

While the assumption that the investor's portfolio choices affect only the drift of the securities' price process (and not also its volatility) may appear restrictive, this can be justified by thinking of such portfolio problem as embedded into the equilibrium problem for an economy with financial securities (i.e., with securities that represents "side bets" between the agents rather than claims to an exogenously given dividend process). For such securities it is well known that the equilibrium conditions only determine the relative risk premium process, but not the drift and the diffusion coefficients separately (see, e.g., Karatzas, Lehoczky and Shreve (1990)): therefore, markets can be cleared by fixing the volatility matrix and only adjusting the drift coefficient in response to the actions of the "large" investor. The assumption that the volatility of the available technologies is independent of the quantities invested is also common in the theory of optimal investment with nonlinear production technologies (see, e.g., Sundaresan (1984)).

\section{The Economic Setting}

We consider a continuous-time economy on the finite time span $[0, T]$, in which an individual endowed with some initial wealth and a stochastic income stream chooses an optimal consumption and investment policy.

Information structure. The uncertainty is represented by a filtered probability space $(\Omega, \mathcal{F}, \mathbf{F}, P)$, on which is defined a $n$-dimensional Brownian motion

$$
w=\left\{\left(w_{1}(t), \ldots, w_{n}(t)\right)^{\top}: t \in[0, T]\right\} .
$$

The filtration $\mathbf{F}=\left\{\mathcal{F}_{t}\right\}$ is the augmentation under $P$ of the filtration generated by $w .^{1}$ We assume that $\mathcal{F}=\mathcal{F}_{T}$, or that the true state of nature is completely determined by the

\footnotetext{
${ }^{1}$ The augmented Brownian filtration $\mathbf{F}=\left\{\mathcal{F}_{t}\right\}$ is defined by $\mathcal{F}_{t}=\sigma\left(\mathcal{F}_{t}^{w} \cup \mathcal{Z}\right)$, where $\mathcal{F}_{t}^{w}=\sigma(w(\tau)$ : $\tau \in[0, t])$ is the smallest sigma-field with respect to which $w(\tau)$ is measurable for every $\tau \in[0, t]$ and $\mathcal{Z}=\{E \subset \Omega: \exists G \in \mathcal{F}$ with $E \subseteq G, P(G)=0\}$ denotes the set of $P$-null events. It is well known that the augmented filtration is continuous and that $w$ is still a Brownian motion with respect to it (Karatzas and Shreve (1988), Corollary 2.7.8 and Propasition 2.7.9).
} 
sample paths of $w$ on $[0, T]$. We interpret the sigma-field $\mathcal{F}_{t}$ as representing the information of the individual at time $t$ and the probability measure $P$ as representing his beliefs. All the stochastic processes to appear in the sequel are progressively measurable with respect to $\mathbf{F}$ and all the equalities involving random variables are understood to hold $P$-a.s.. ${ }^{2}$

Consumption space. There is a single perishable good (the numeraire). The consumption space $\mathcal{C}$ is given by the set of adapted consumption rate processes $c$ with $\int_{0}^{T}|c(t)| d t<\infty$. The individual consumption set will be shortly specified as a subset of the non-negative orthant $\mathcal{C}_{+}$.

Securities market. The investment opportunities are represented by $n+1$ long-lived securities. The first security (the "bond") is a locally riskless savings account earning the instantaneous interest rate process $r$, so that its value process $B$ evolves according to

$$
B(t, \omega)=B(0)+\int_{0}^{t} r(\tau, \omega) B(\tau, \omega) d \tau .
$$

The remaining $n$ assets are risky. Letting $S=\left(S_{1}, \ldots, S_{n}\right)$ denote their price process and $D=\left(D_{1}, \ldots, D_{n}\right)$ their cumulative dividend process, we assume that $S+D$ is an Itô process:

$$
S(t, \omega)+D(t, \omega)=S(0)+\int_{0}^{t} I_{S}(\tau, \omega) \mu(\tau, \omega) d \tau+\int_{0}^{t} I_{S}(\tau, \omega) \sigma(\tau, \omega) d w(\tau, \omega),
$$

where $I_{S}(t)$ denotes the $n \times n$ diagonal matrix with elements $S(t)$.

We allow explicit dependence of the price process for the traded securities on the portfolio strategy chosen by the investor by assuming that

$$
r(t, \omega)=\bar{r}(t, \omega)+\breve{r}(\alpha(t, \omega), \theta(t, \omega), t, \omega),
$$

and

$$
\mu(t, \omega)=\bar{\mu}(t, \omega)+\breve{\mu}(\alpha(t, \omega), \theta(t, \omega), t, \omega)
$$

for some functions $\breve{r}: \mathbb{R} \times \mathbf{R}^{n} \times[0, T] \times \Omega \rightarrow \overline{\mathbb{R}}$ and $\breve{\mu}: \mathbb{R} \times \mathbb{R}^{n} \times[0, T] \times \Omega \rightarrow \overline{\mathbb{R}}^{n}$, where $\alpha$ and $\theta=\left(\theta_{1}, \ldots, \theta_{n}\right)$ denote, respectively, the dollar amounts invested in the bond and in the $n$ risky asset. Without loss of generality, we assume that $\breve{r}(0,0, t, \omega)=0$ and $\breve{\mu}(0,0, t, \omega)=0$ for all $(t, \omega) \in[0, T] \times \Omega$. Clearly, the case $\breve{r} \equiv 0$ and $\breve{\mu} \equiv 0$ corresponds to the usual setting with exogenously fixed prices. In the sequel we will suppress the explicit dependence on the state $\omega$ whenever no possibility of confusion arises.

Assumption 1. The process $\bar{r}$ satisfies

$$
\int_{0}^{T} \bar{r}(t)^{-} d t<K_{r}
$$

for some $K_{r}>0$, where $x^{-}=\max (0,-x)$ denotes the negative part of the real number $x$.

${ }^{2} \mathrm{~A}$ process $X=\{X(t): t \in[0, T]\}$ is said to be progressively measurable with respect to the filtration $\mathbf{F}=\left\{F_{t}\right\}$ if for every $t \in[0, T]$, the map $(s, \omega) \mapsto X(s, \omega)$ from $\left(\{0, t] \times \Omega, \mathcal{B}([0, t]) \otimes \mathcal{F}_{t}\right)$ into $\left(\mathbb{R}^{n}, B\left(\mathbb{R}^{n}\right)\right)$ is measurable, where $\mathcal{B}([0, t]) \otimes \mathcal{F}_{t}$ denotes the product $\sigma$-field of the Borel $\sigma$-field on $[0, t]$ and $\mathcal{F}_{t}$, and $\mathcal{B}\left(\mathbb{R}^{n}\right)$ denotes the Borel $\sigma$-field on $\mathbb{R}^{n}$. Recalling that a process $X$ is adapted to $\mathrm{F}$ if $X(t)$ is an $\mathcal{F}_{t}$-measurable random variable for all $t \in[0, T]$, we have that every progressively measurable process is adapted. Conversely, any adapted process with right- or left-continuous paths is progressively measurable. 
The above assumption is in particular satisfied if $\bar{r}$ is nonnegative or bounded below.

Assumption 2. The diffusion matrix $\sigma(t)$ satisfies the nondegeneracy condition

$$
x^{\top} \sigma(t) \sigma(t)^{\top} x \geq \varepsilon|x|^{2}
$$

almost surely for all $(x, t) \in \mathbb{R}^{n} \times[0, T]$ and some $\varepsilon>0$. Moreover, letting

$$
\kappa_{0}=-\sigma^{-1}(\bar{\mu}-\bar{r} \overline{1})
$$

where $\overline{\mathbb{1}}=(1, \ldots, 1)^{\top} \in \mathbb{R}^{n}$, we have

$$
\mathrm{E}\left[\exp \left(\frac{1}{2} \int_{0}^{T}\left|\kappa_{0}(t)\right|^{2} d t\right)\right]<\infty
$$

Condition (4) implies in particular that $\sigma(t)$ has full rank a.s. for all $t \in[0, T]$, so that, in the absence of feedback effects on prices, markets would be dynamically complete, and that $\sigma(t, \omega)^{-1}$ has essentially bounded matrix norm, uniformly in $(t, \omega) \in[0, T] \times \Omega$ (Karatzas and Shreve (1988), Problem 5.8.1). Condition (6) is used to guarantee the existence of an equivalent martingale measure.

Trading strategies. Trading takes place continuously and there are no market frictions. An admissible trading strategy is a $(n+1)$-dimensional vector process $(\alpha, \theta)$ satisfying

$$
\begin{gathered}
\int_{0}^{T}\left|\alpha(t)(\bar{r}(t)+\check{r}(\alpha(t), \theta(t), t))+\theta(t)^{\top}(\bar{\mu}(t)+\breve{\mu}(\alpha(t), \theta(t), t))\right| d t \\
+\int_{0}^{T}\left|\theta(t)^{\top} \sigma(t)\right|^{2} d t<\infty .
\end{gathered}
$$

The set of admissible trading strategies is denoted by $\Theta$.

Preferences and endowments. Preferences for the individual are represented by a timeadditive utility function ${ }^{3}$

$$
U(c)=E\left[\int_{0}^{T} u(c(t), t) d t\right],
$$

which is well defined for all consumption processes $c \in \mathcal{C}_{+}$satisfying

$$
\mathrm{E}\left[\int_{0}^{T} u(c(t), t)^{-} d t\right]<\infty
$$

The individual consumption set, denoted by $\mathcal{C}_{+}^{*}$, will be accordingly given by the consumption processes $c \in \mathcal{C}_{+}$satisfying (9).

Assumption 3. The function $u(\cdot, t)$ is increasing, strictly concave and continuously differentiable on $(0, \infty)$ for all $t \in[0, T]$. Moreover, it satisfies the Inada conditions

$$
\lim _{c \downarrow 0} u_{c}(c, t)=\infty \quad \text { and } \quad \lim _{c \uparrow \infty} u_{c}(c, t)=0
$$

\footnotetext{
${ }^{3}$ The case in which preferences include a bequest function $V(W)$ for final wealth is not substantially different and all the results in this paper apply, provided that $V(\cdot)$ satisfies the same conditions placed on $u(\cdot, t)$.
} 
and there exist constants $\delta \in(0,1)$ and $\gamma \in(0, \infty)$ such that

$$
\delta u_{c}(c, t) \geq u_{c}(\gamma c, t) \quad \forall(c, t) \in(0, \infty) \times[0, T] .
$$

Finally, $u(c, \cdot)$ is continuous and decreasing on $[0, T]$ for all $c>0$.

Remark 1. Condition (10) is well understood and it implies in particular that the derivative function $u_{c}(\cdot, t)$ has a continuous and strictly decreasing inverse $f(\cdot, t)$ mapping $(0, \infty)$ onto itself. Condition (11) has the purpose of guaranteeing that certain functionals to be introduced in the sequel can be differentiated under the integral sign. It is easily verified that this condition holds for the utility functions $u(c, t)=\rho(t) \log c$ or $u(c, t)=\rho(t) \frac{c^{1-b}}{1-b}$, $b>0, b \neq 1$. Also, taking $c=f(y, t)$ in (11), applying $f(\cdot, t)$ to both sides and iterating shows that the following property holds

$$
\forall \delta \in(0, \infty), \exists \gamma \in(0, \infty) \text { such that } f(\delta y, t) \leq \gamma f(y, t), \forall(y, t) \in(0, \infty) \times[0, T] .
$$

The agent is endowed with some initial wealth $W_{0} \geq 0$ and a bounded stochastic income stream $y \in \mathcal{C}_{+}^{*}$.

\section{Feasible consumption processes}

A consumption process $c \in \mathcal{C}_{+}^{*}$ is said to be feasible if there exists an admissible trading strategy $(\alpha, \theta) \in \Theta$ such that letting

$$
W(t)=\alpha(t)+\sum_{k=1}^{n} \theta_{k}(t)
$$

denote the value of the agent's portfolio at time $t$, we have

$$
\begin{gathered}
W(t)=W_{0}+\int_{0}^{t}\left[\alpha(\tau) \bar{r}(\tau)+\theta(\tau)^{\top} \bar{\mu}(\tau)+g(\alpha(\tau), \theta(\tau), \tau)\right] d \tau \\
+\int_{0}^{t} \theta(\tau)^{\top} \sigma(\tau) d w(\tau)-\int_{0}^{t}(c(\tau)-y(\tau)) d \tau \\
W(t) \geq-K \exp \left(\int_{0}^{t} \bar{r}(\tau) d \tau\right) \\
W(T) \geq 0
\end{gathered}
$$

for all $t \in[0, T]$ and some $K \in \mathbf{R}_{+}$, where

$$
g(\alpha, \theta, t, \omega)=\alpha \breve{r}(\alpha, \theta, t, \omega)+\theta^{\top} \breve{\mu}(\alpha, \theta, t, \omega) .
$$

If the above conditions are satisfied, the trading strategy $(\alpha, \theta)$ is said to finance $c$.

Equation (13) is the usual dynamic budget constraint: it states that the wealth at any time $t \in[0, T]$ equals the initial wealth, plus the trading gains, minus the cumulative net consumption. The only difference from the standard setting is that the stochastic integral equation for the wealth process is non-linear in the trading strategy $(\alpha, \theta)$ : this non-linearity arises from the effect of trading strategies on prices and is captured by the function $g$. 
Equation (15) states that, while the investor is allowed to borrow against future income and thus to have short-term deficits, the final wealth must be non-negative: in other words, final wealth must be sufficient to cover any amount borrowed. Moreover, the discounted wealth process is required to admit a uniform lower bound (equation (14)): in the standard setting where $g=0$, it is well known (from Dybvig and Huang (1989)) that this condition is sufficient to rule out arbitrage opportunities, such as the doubling strategies discussed by Harrison and Kreps (1979). We will verify later that the same is true in the current setting (see Lemma 1). 4

The following additional assumption will be maintained throughout the paper.

Assumption 4. The function $g$ of $(16)$ is concave and upper semicontinuous in $(\alpha, \theta)$ and bounded above on $\mathbb{R} \times \mathbb{R}^{n} \times[0, T] \times \Omega$.

The requirement that $g$ be bounded above and concave has an obvious economic interpretation: the instantaneous expected return on the portfolio of the "large" investor is adversely affected by his trades (so that typically $g(\alpha, \theta, t) \leq g(0,0, t)=0$ ), and larger trades have a proportionally larger impact on the portfolio's expected return.

Next, for $\nu=\left(\nu_{0}, \nu_{-}\right) \in \mathbb{R} \times \mathbb{R}^{n}$, let

$$
\begin{aligned}
\tilde{g}(\nu, t, \omega) & =\sup _{(\alpha, \theta) \in \mathbb{R} \times \mathbb{R}^{n}} g(\alpha, \theta, t, \omega)-\alpha \nu_{0}-\theta^{\top} \nu_{-} \\
& =\sup _{(\alpha, \theta) \in \mathbb{R} \times \mathbf{R}^{n}} \alpha\left(\breve{r}(\alpha, \theta, t, \omega)-\nu_{0}\right)+\theta^{\top}\left(\breve{\mu}(\alpha, \theta, t, \omega)-\nu_{-}\right)
\end{aligned}
$$

denote the convex conjugate of $-g(-\alpha,-\theta, t, \omega)$ and let $\mathcal{N}_{t}(\omega)$ denote the effective domain of $\tilde{g}(\cdot, t, \omega)$ :

$$
\mathcal{N}_{t}(\omega)=\left\{\nu \in \mathbb{R}^{n+1}: \tilde{g}(\nu, t ; \omega)<\infty\right\}
$$

We remark that $\tilde{g}$ is a nonnegative, convex, lower semicontinuous function (Rockafellar (1974), Theorem 5) and $\mathcal{N}_{t}(\omega)$ is a nonempty, closed convex set with $0 \in \mathcal{N}_{t}(\omega)$ for all $(t, \omega) \in[0, T] \times \Omega$. Denote by $\mathcal{N}$ the family of $n+1$-dimensional adapted processes $\nu$ such that $\nu(t, \omega) \in \mathcal{N}_{t}(\omega)$ for all $(t, \omega) \in[0, T] \times \Omega$. Clearly, $\mathcal{N}$ is non-empty, since, at least, $0 \in \mathcal{N}$. We will need the following assumption, which is satisfied in all the examples below except for the case of portfolio constraints.

Assumption 5. The function $\bar{g}$ is bounded on its effective domain. Moreover, the sets $\mathcal{N}_{t}(\omega)$ are uniformly bounded.

It is easily verified that a sufficient condition for the last part of the assumption to be satisfied is that $g(\cdot, t, \omega)$ be uniformly Lipschitz, i.e., that

$$
\left|g\left(\alpha_{1}, \theta_{1}, t, \omega\right)-g\left(\alpha_{2}, \theta_{2}, t, \omega\right)\right| \leq K\left(\left|\alpha_{1}-\alpha_{2}\right|+\left|\theta_{1}-\theta_{2}\right|\right)
$$

for some $K>0$ and all $\left(\alpha_{1}, \theta_{1}\right),\left(\alpha_{2}, \theta_{2}\right) \in \mathbb{R}^{n+1}$ and $(t, \omega) \in[0, T] \times \Omega$.

\footnotetext{
${ }^{4}$ In a discrete time economy, Jarrow $(1992,1994)$ has investigated the existence of arbitrage opportunities (which he calls market manipulation trading strategies) for an investor whose trades affect prices and provided sufficient conditions for their nonexistence.
} 
Remark 2. It follows from Theorem 5 in Rockafellar (1974) that

$$
g(\alpha, \theta, t, \omega)=\inf _{\nu \in \mathcal{N}_{t}(\omega)}\left[\tilde{g}(\nu, t, \omega)+\alpha \nu_{0}+\theta^{\top} \nu_{-}\right]
$$

By the compactness of $\mathcal{N}_{t}(\omega)$ and the lower semicontinuity of $\tilde{g}$, this implies that for any $(\alpha, \theta) \in \theta$ there exists a $\nu \in \mathcal{N}$ such that

$$
g(\alpha(t), \theta(t), t)=\tilde{g}(\nu(t), t)+\alpha(t) \nu_{0}(t)+\theta(t)^{\top} \nu_{-}(t)
$$

\section{Examples}

The following examples motivate the analysis in the present paper and illustrate the connection with the optimal consumption problem with constraints on the investment policies. For purposes of comparison, we start from the standard complete-market setting in which prices are exogenously fixed.

Standard setting: In this case $\breve{r} \equiv 0$ and $\breve{\mu} \equiv 0$, which implies $g \equiv 0$ and

$$
\tilde{g}(\nu, t, \omega)= \begin{cases}0 & \text { if } \nu=0 \\ \infty & \text { otherwise }\end{cases}
$$

Therefore

$$
\mathcal{N}_{t}(\omega)=\{0\} \text { for all }(t, \omega) \in[0, T] \times \Omega .
$$

This is the setting examined by Karatzas, Lehoczky and Shreve (1987) and Cox and Huang $(1989,1991)$.

Price pressure: Let $\breve{r} \equiv 0$ and

$$
\breve{\mu}(\alpha, \theta, t, \omega)= \begin{cases}-\frac{a(t, \omega) \theta}{|\theta|} & \text { if } \theta \neq 0 ; \\ 0 & \text { otherwise }\end{cases}
$$

for some nonnegative bounded process $a$, so that buying a risky asset depresses its expected return, while shorting it increases the expected return. In this case it is easily verified that

$$
g(\alpha, \theta, t, \omega)=-a(t, \omega)|\theta|
$$

(the expected return on wealth decreases in a concave fashion with the absolute amounts invested in risky assets) and

$$
\bar{g}(\nu, t, \omega)= \begin{cases}0 & \text { if } \nu_{0}=0 \text { and }|\nu| \leq a(t, \omega) \\ \infty & \text { otherwise }\end{cases}
$$

Therefore,

$$
\mathcal{N}_{t}(\omega)=\left\{\nu \in \mathbb{R}^{n+1}: \nu_{0}=0 \text { and }|\nu| \leq a(t, \omega)\right\}
$$

More generally, if

$$
\breve{\mu}(\alpha, \theta, t, \omega)= \begin{cases}-\frac{A(t, \omega) \theta}{|\theta|} & \text { if } \theta \neq 0 ; \\ 0 & \text { otherwise }\end{cases}
$$


where $A$ is a positive-definite bounded matrix process, then

$$
g(\alpha, \theta, t, \omega)= \begin{cases}-\frac{\theta^{\top} A(t, \omega) \theta}{|\theta|} & \text { if } \theta \neq 0 \\ 0 & \text { otherwise }\end{cases}
$$

and

Therefore,

$$
\tilde{g}(\nu, t, \omega)= \begin{cases}0 & \text { if } \nu_{0}=0 \text { and }\left|A(t, \omega)^{-1} \nu\right| \leq 1 \\ \infty & \text { otherwise. }\end{cases}
$$

$$
\mathcal{N}_{t}(\omega)=\left\{\nu \in \mathbb{R}^{n+1}: \nu_{0}=0 \text { and }\left|A(t, \omega)^{-1} \nu\right| \leq 1\right\}
$$

Different interest rates for borrowing and lending: Suppose that the borrowing rate $\bar{R}$ is not necessarily equal to the lending rate $\bar{r}$, and $\bar{r} \leq \bar{R}$. We can model this by setting $\breve{\mu} \equiv 0$ and

$$
\breve{r}(\alpha, \theta, t, \omega)=(\bar{R}(t, \omega)-\bar{r}(t, \omega)) \mathbf{1}_{\{\alpha<0\}},
$$

where $\mathbf{1}_{\{\alpha<0\}}=1$ if $\alpha<0$ and $1_{\{\alpha<0\}}=0$ otherwise. Then

$$
g(\alpha, \theta, t, \omega)=\alpha(\bar{R}(t, \omega)-\bar{r}(t, \omega)) \mathbf{1}_{\{\alpha<0\}}
$$

It is easy to see that

$$
\mathcal{N}_{t}(\omega)=\left\{\nu \in \mathbb{R}^{n+1}: 0 \leq \nu_{0} \leq \bar{R}(t, \omega)-\bar{r}(t, \omega) \text { and } \nu_{-}=0\right\}
$$

and that $\tilde{g}(\cdot, t, \omega) \equiv 0$ on $\mathcal{N}_{t}(\omega)$, for all $(t, \omega) \in[0, T] \times \Omega$.

Portfolio constraints: Let $K$ be a nonempty, closed, convex set in $\mathbb{R}^{n+1}$ and let $1_{K}$ denote its indicator function in the sense of convex analysis, namely $1_{K}((\alpha, \theta))=0$ if $(\alpha, \theta) \in K$ and $1_{K}((\alpha, \theta))=\infty$ otherwise. Let

$$
\breve{r}(\alpha, \theta, t, \omega)= \begin{cases}-\frac{\alpha}{|\alpha|^{2}} 1_{K}((\alpha, \theta)) & \text { if } \alpha \neq 0 ; \\ 0 & \text { otherwise }\end{cases}
$$

and

$$
\breve{\mu}(\alpha, \theta, t, \omega)= \begin{cases}-\frac{\theta}{|\theta|^{2}} 1_{K}((\alpha, \theta)) & \text { if } \theta \neq 0 ; \\ 0 & \text { otherwise, }\end{cases}
$$

so that taking a position in any traded assets is always "infinitely bad", unless $(\alpha, \theta) \in K$. Clearly, this setting is equivalent to having the price process to be independent of the investor's portfolio choice but constraining $(\alpha, \theta)$ to take values in $K$. In this case, we have

$$
g(\alpha, \theta, t, \omega)=-1_{K}((\alpha, \theta))
$$

and

$$
\tilde{g}(\nu, t, \omega)=\sup _{(\alpha, \theta) \in K}-\left(\alpha \nu_{0}+\theta^{\top} \nu_{-}\right)=\delta_{-K}(\nu),
$$

where $\delta_{-K}$ denotes the support function of the convex set $-K$. In this case

$$
\mathcal{N}_{t}(\omega)=\left\{\nu \in \mathbb{R}^{n+1}: \delta_{-K}(\nu)<\infty\right\}
$$


is the barrier cone of $-K$. This problem with convex constraints on the investment strategies was dealt with by Cuoco (1995), building on previous work by He and Pearson (1991), Karatzas, Lehoczky, Shreve and Xu (1991), Xu and Shreve (1992a), and Cvitanić and Karatzas $(1992,1993)$. We remark that this setting is not included in our analysis, since the sets $\mathcal{N}_{t}(\omega)$ are convex cones, and hence not bounded. Nevertheless, the feasibility (hedging) result of Theorem 1 below, and the subsequent remark, are still valid, as shown in the above mentioned literature, provided that free disposal of wealth is allowed.

\section{No-Arbitrage State-Price Densities}

Since security prices and the individual income stream are allowed to be possibly nonMarkovian processes, dynamic programming techniques cannot be applied to analyze the individual consumption problem. Therefore we will derive a martingale characterization of the optimal policies using the duality techniques developed by He and Pearson (1991), Karatzas, Lehoczky, Shreve and Xu (1991), He and Pagès (1991) and Cvitanić and Karatzas (1992) to analyze the optimal consumption problem with constrained investment policies. In order to formulate the proper dual shadow state-price problem, we start by identifying the set of state-price densities for the economy consistent with the absence of arbitrage opportunities. 5

For an arbitrary process $\nu \in \mathcal{N}$, define the exponential martingale

$$
\xi_{\nu}(t)=\exp \left(\int_{0}^{t} \kappa_{\nu}(\tau)^{\top} d w(\tau)-\frac{1}{2} \int_{0}^{t}\left|\kappa_{\nu}(\tau)\right|^{2} d \tau\right)
$$

and the discount factor

$$
\beta_{\nu}(t)=\exp \left(-\int_{0}^{t}\left(\bar{r}(\tau)+\nu_{0}(\tau)\right) d \tau\right)
$$

where

$$
\kappa_{\nu}(t)=-\sigma(t)^{-1}\left(\bar{\mu}(t)+\nu_{-}(t)-\left(\bar{r}(t)+\nu_{0}(t)\right) \overline{1}\right)
$$

and let

$$
\pi_{\nu}(t) \doteq \beta_{\nu}(t) \xi_{\nu}(t)
$$

Clearly, each $\pi_{\nu}$ with $\nu \in \mathcal{N}$ would represent the unique state-price density in a shadow economy in which the portfolio policy $(\alpha, \theta)$ of the "large" investor was known to be such that $\breve{r}(\alpha(t), \theta(t), t)=\nu_{0}(t)$ and $\breve{\mu}(\alpha(t), \theta(t), t)=\nu_{-}(t)$ for all $t \in[0, T]$. More generally, the following lemma shows that any process $\pi_{\nu}$ with $\nu \in \mathcal{N}$ can be interpreted as a shadow state-price density for the economy.

Lemma 1. If $\mathrm{c} \in \mathcal{C}_{+}^{*}$ is a feasible consumption process, then

$$
\mathrm{E}\left[\int_{0}^{T} \pi_{\nu}(t)(c(t)-y(t)) d t\right] \leq W_{0}+\mathrm{E}\left[\int_{0}^{T} \pi_{\nu}(t) \tilde{g}(\nu(t), t) d t\right]
$$

holds for all $\nu \in \mathcal{N}$.

\footnotetext{
${ }^{5}$ In our setting, an arbitrage opportunity is a nonzero consumption process $c \in \mathcal{C}_{+}^{*}$ that is feasible with zero initial wealth and zero income.
} 
Proof. Using (13) and Itô's lemma, it is easy to show that

$$
\begin{gathered}
\pi_{\nu}(t) W(t)+\int_{0}^{t} \pi_{\nu}(\tau)(c(\tau)-y(\tau)) d \tau-\int_{0}^{t} \pi_{\nu}(\tau)\left(\theta(\tau)^{\top} \sigma(\tau)+W(\tau) \kappa_{\nu}(\tau)^{\tau}\right) d w(\tau) \\
=W_{0}+\int_{0}^{t} \pi_{\nu}(\tau) \\
\left(g(\alpha(\tau), \theta(\tau), \tau)-\alpha(\tau) \nu_{0}(\tau)-\theta(\tau)^{\top} \nu_{-}(\tau)\right) d \tau \\
\leq W_{0}+\int_{0}^{t} \pi_{\nu}(\tau) \tilde{g}(\nu(\tau), \tau) d \tau
\end{gathered}
$$

holds for all $\nu \in \mathcal{N}$, where the inequality follows from the definition of $\tilde{g}$. For each positive integer $n$, let

$$
\tau_{n}=T \wedge \inf \left\{t \in[0, T]: \int_{0}^{t}\left|\pi_{\nu}(\tau)\left(\theta(\tau)^{\top} \sigma(\tau)+W(\tau) \kappa_{\nu}(\tau)^{\top}\right)\right|^{2} d \tau \geq n\right\},
$$

with the usual convention, maintained for the remainder of the paper, that $\inf (\emptyset)=\infty$. Since the stochastic integral in (22) is a martingale on $\left[0, \tau_{n}\right]$, taking expectations gives

$$
\mathrm{E}\left[\pi_{\nu}\left(\tau_{n}\right) W\left(\tau_{n}\right)\right]+\mathrm{E}\left[\int_{0}^{\tau_{n}} \pi_{\nu}(t)(c(t)-y(t)) d t\right] \leq W_{0}+\mathrm{E}\left[\int_{0}^{\tau_{n}} \pi_{\nu}(t) \bar{g}(\nu(t), t) d t\right] .
$$

Letting $n \uparrow \infty$, we have $\tau_{n} \uparrow T$ (because of (7) and the continuity of $\pi_{\nu}$ and $W$ ). Applying the monotone convergence theorem twice and using the fact that $\mathrm{E}\left[\int_{0}^{T} \pi_{\nu}(t) y(t) d t\right]$ is finite under our assumptions, shows that

$$
\lim _{n \rightarrow \infty} \mathrm{E}\left[\int_{0}^{\tau_{n}} \pi_{\nu}(t)(c(t)-y(t)) d t\right]=\mathrm{E}\left[\int_{0}^{T} \pi_{\nu}(t)(c(t)-y(t)) d t\right]
$$

By the same argument,

$$
\lim _{n \rightarrow \infty} \mathrm{E}\left[\int_{0}^{\tau_{n}} \pi_{\nu}(t) \bar{g}(\nu(t), t) d t\right]=\mathrm{E}\left[\int_{0}^{T} \pi_{\nu}(t) \tilde{g}(\nu(t), t) d t\right] .
$$

As for the first term in (23), we have from (14)

$$
\left(\pi_{\nu}\left(\tau_{n}\right) W\left(\tau_{n}\right)\right)^{-} \leq K \exp \left(-\int_{0}^{\tau_{n}} \nu_{0}(t) d t\right) \xi_{\nu}\left(\tau_{n}\right)
$$

Since the family of random variables $\left\{\xi_{\nu}(t): t \in[0, T]\right\}$ is bounded in $L^{2}(P)$ and $\nu_{0}$ is bounded, Fatou's lemma for random variables uniformly integrable from below (Chow and Teicher (1988), Theorem 4.2.2) gives

$$
\liminf _{n \rightarrow \infty} \mathrm{E}\left[\pi_{\nu}\left(\tau_{n}\right) W\left(\tau_{n}\right)\right] \geq \mathrm{E}\left[\pi_{\nu}(T) W(T)\right] \geq 0,
$$

where the last inequality follows from (15). This establishes (21). 
By the above lemma, the fact that $0 \in \mathcal{N}$ is sufficient to rule out the existence of arbitrage opportunities. We will refer to (21) as a static budget constraint. Karatzas, Lehoczky and Shreve (1987) and Cox and Huang (1991) have shown that, in the standard setting where $\breve{r} \equiv 0, \breve{\mu} \equiv 0$ and $\mathcal{N}=\{0\}$, a consumption process is feasible if and only if it satisfies a static budget constraint with respect to the unique state-price density $\pi_{0}$. The following theorem gives a general version of this result by showing that the satisfaction of a budget constraint with respect to each $\pi_{\nu}$ with $\nu \in \mathcal{N}$ is sufficient to guarantee feasibility in our setting.

Theorem 1. Let $c \in \mathcal{C}_{+}^{*}$ be a consumption process and suppose that there exists a process $\nu^{*} \in \mathcal{N}$ such that for all $\nu \in \mathcal{N}:$

$\mathrm{E}\left[\int_{0}^{T} \pi_{\nu}(t)(c(t)-y(t)-\tilde{g}(\nu(t), t)) d t\right] \leq \mathrm{E}\left[\int_{0}^{T} \pi_{\nu^{*}}(t)\left(c(t)-y(t)-\tilde{g}\left(\nu^{*}(t), t\right)\right) d t\right]=W_{0}$.

Then $c$ is feasible and the optimal wealth process is given by

$$
W_{\nu^{*}}(t)=\pi_{\nu^{*}}(t)^{-1} \mathrm{E}\left[\int_{t}^{T} \pi_{\nu^{*}}(\tau)\left(c(\tau)-y(\tau)-\tilde{g}\left(\nu^{*}(\tau), \tau\right)\right) d t \mid \mathcal{F}_{t}\right] .
$$

Proof. See Appendix A.

Remark 3. In fact, using the methods of El Karoui, Peng and Quenez (1995) or Cvitanić and Karatzas (1993), it is possible to show a stronger result: the minimal initial wealth $W_{0}$ required for financing a given consumption process $c$ and a given terminal wealth $W$, assuming the endowment stream $y$, is given by

$$
W_{0}=\sup _{\nu \in \mathcal{N}} \mathrm{E}\left[\int_{0}^{T} \pi_{\nu}(t)(c(t)-y(t)-\tilde{g}(\nu(t), t)) d t+\pi_{\nu}(T) W\right] .
$$

\section{Optimal Consumption Policies}

Letting $c^{*}$ denote the optimal consumption policy, Theorem 1 suggests that there should exist a Lagrangian multiplier $\psi^{*}>0$ such that $\left(c^{*}, \psi^{*}, \nu^{*}\right)$ is a saddle point of the map

$$
\mathcal{L}(c, \psi, \nu)=U(c)-\psi\left(\mathrm{E}\left[\int_{0}^{T} \pi_{\nu}(t)(c(t)-y(t)-\tilde{g}(\nu(t), t)) d t\right]-W_{0}\right),
$$

where we maximize with respect to $c$ and minimize with respect to $(\psi, \nu)$.

Let

$$
\tilde{u}(y, t) \equiv \max _{c \geq 0}[u(c, t)-y c]=u(f(y, t), t)-y f(y, t)
$$

denote the convex conjugate of $-u(-c, t)$. The following lemma collects some properties of the function $\tilde{u}$ that will be used repeatedly in the sequel.

Lemma 2. The function $\tilde{u}(\cdot, t):(0, \infty) \rightarrow \mathbb{R}$ is strictly decreasing and strictly convex for all $t \in[0, T]$, with $\frac{\partial}{\partial y} \tilde{u}(y, t)=-f(y, t)$. Moreover

$$
\tilde{u}(0+, t)=u(\infty, t), \quad \tilde{u}(\infty, t)=u(0+, t) .
$$

Proof. See, e.g., Karatzas, Lehoczky, Shreve and Xu (1991), p. 707. 
Maximization of (26) with respect to $c$ gives

$$
J(\psi, \nu)=\mathrm{E}\left[\int_{0}^{T} \tilde{u}\left(\psi \pi_{\nu}(t), t\right) d t+\psi \int_{0}^{T} \pi_{\nu}(t)(y(t)+\tilde{g}(\nu(t), t)) d t\right]+\psi W_{0},
$$

where we remark that the above expectation is well defined for all $(\psi, \nu) \in(0, \infty) \times \mathcal{N}$, since from the inequality

$$
u(1, t)-y \leq \max _{c>0}[u(c, t)-y c]=u(f(y, t), t)-y f(y, t),
$$

we have

$$
\mathrm{E}\left[\int_{0}^{T} \tilde{u}\left(\psi \pi_{\nu}(t), t\right)^{-} d t\right] \leq \int_{0}^{T} u(1, t) d t+\psi \mathrm{E}\left[\int_{0}^{T} \pi_{\nu}(t) d t\right]<\infty
$$

(where the last inequality follows from Assumption 3, (3) and the martingale property of $\left.\xi_{\nu}\right)$. Therefore $J:(0, \infty) \times \mathcal{N} \rightarrow \mathbb{R} \cup\{\infty\}$ and we are left with the shadow state-price problem

$$
\min _{(\psi, \nu) \in(0, \infty) \times \mathcal{N}} J(\psi, \nu)
$$

The following theorem establishes the duality between the individual's constrained optimization problem and $\left(P^{*}\right)$.

Theorem 2. Assume that $\left(\psi^{*}, \nu^{*}\right) \in(0, \infty) \times \mathcal{N}$ solves the shadow state-price problem $\left(P^{*}\right)$ and

$$
\mathrm{E}\left[\int_{0}^{T} \pi_{\nu^{*}}(t) f\left(\psi^{*} \pi_{\nu^{*}}(t), t\right) d t\right]<\infty
$$

Then the policy

$$
c_{\nu^{*}}(t)=f\left(\psi^{*} \pi_{\nu^{*}}(t), t\right)
$$

is optimal and the optimal wealth process is given by (25) with $c=c_{\nu^{*}}$. Moreover, the optimal investment strategy $(\alpha, \theta)$ satisfies

$$
g(\alpha(t), \theta(t), t)=\tilde{g}\left(\nu^{*}(t), t\right)+\alpha(t) \nu_{0}^{*}(t)+\theta(t)^{\top} \nu_{-}^{*}(t), \quad(\lambda \times P) \text {-a.e. }
$$

Proof. See Appendix A.

We can interpret the optimal policy $c_{\nu^{*}}$ as the optimal policy in a standard economy with price coefficients $\mathcal{P}_{\nu^{*}}=\left(\bar{r}+\nu_{0}^{*}, \bar{\mu}+\nu_{-}^{*}, \sigma\right)$ and endowment process $y(t)+\tilde{g}\left(\nu^{*}(t), t\right)$, as $c_{\nu^{*}}$ also solves the problem

$$
\begin{gathered}
\max _{c \in \mathcal{C}_{+}^{*}} U(c) \\
\text { s.t. } \mathrm{E}\left[\int_{0}^{T} \pi_{\nu^{*}}(t)\left(c(t)-y(t)-\tilde{g}\left(\nu^{*}(t), t\right) d t\right] \leq W_{0} .\right.
\end{gathered}
$$

In fact, (32) shows that $\tilde{g}\left(\nu^{*}(t), t\right)+\alpha(t) \nu_{0}^{*}(t)+\theta(t)^{\top} \nu_{-}^{*}(t)$ represents the change at time $t$ in the instantaneous expected return of the optimally invested wealth induced by the investor's portfolio choice. Following the terminology introduced by He and Pearson, we will refer to $\xi_{\nu^{*}}$ as the minimax martingale density and to $\pi_{\nu^{*}}$ as the minimax state-price density for the individual. They must clearly be unique when they exist.

The next theorem provides sufficient conditions for the existence of a minimax stateprice density and of an optimal consumption/investment policy. 
Theorem 3. Assume that

(a) $u(\infty, t)=\infty$ for all $t \in[0, T]$ and $u(c, t)^{-} \leq k\left(1+c^{1-b}\right)$ on $(0, \infty) \times[0, T]$ for some $k \geq 0, b \geq 1$;

(b) either $W_{0}>0$ or $y / B>\varepsilon(\lambda \times P)-$ a.e. for some $\varepsilon>0$;

(c) for all $\psi \in(0, \infty)$, there exists a $\nu \in \mathcal{N}$ such that $J(\psi, \nu)<\infty$.

Then the minimum in $\left(P^{*}\right)$ is attained and hence a minimax state-price density exists. If in addition

(d) $c u_{c}(c, t) \leq a+(1-b) u(c, t)$ on $(0, \infty) \times[0, T]$ for some $a \geq 0, b>0$,

then condition (30) of Theorem 2 is also satisfied, and hence there exists a constrained optimal consumption/investment policy.

Proof. See Appendix B.

Remark 4. Since the dual functional $J$ is not convex in $\nu$, existence of a solution to the dual problem is proved in the Appendix by reformulating $\left(P^{*}\right)$ as a minimization problem directly over the set of state-price densities $\pi_{\nu}$ rather than over the set of "Lagrangian multipliers" $\nu$ representing them.

Remark 5. Conditions (a) and (c) of Theorem 3 are in particular satisfied if either $u(c, t)$ is bounded below on $(0, \infty) \times[0, T]$ and $u(\infty, t)=\infty$ for all $t \in[0, T]$, or $u(c, t)=\rho(t) \log c$ for some bounded measurable function $\rho:[0, T] \mapsto(0, \bar{\rho}]$. Also, since $0 \in \mathcal{N}$, a sufficient condition for assumption (c) is that $J(\psi, 0)<\infty$ for all $\psi \in(0, \infty)$. In particular, if $u(c, t)$ is nonnegative and satisfies the growth condition $u(c, t) \leq k\left(1+c^{1-b}\right)$ for some $k>0$, $b \in(0,1)$, then (c) will also hold (cf. Karatzas, Lehoczky, Shreve and Xu (1991), Remark 11.9).

Remark 6. Proceeding as in Cuoco (1995), it would have also been possible to establish the existence of an optimal consumption policy without resorting to duality. However, we will see in the next sections that the dual problem offers computational advantages.

\section{Explicit Solution for a Logarithmic Investor}

Suppose that $u(c, t)=e^{-\rho t} \log (c)$ and that $y \equiv 0$, so that the investor is only endowed with some positive amount of wealth $W_{0}$. Also, assume that $\tilde{g} \equiv 0$ on its effective domain. Then we have

$$
\tilde{u}(y, t)=\max _{c \geq 0}\left[e^{-\rho t} \log (c)-y c\right]=-e^{-\rho t}(1+\rho t+\log (y)),
$$

and the dual problem becomes

$$
\begin{array}{r}
\min _{(\psi, \nu) \in(0, \infty) \times \mathcal{N}} \mathrm{E}\left[-\int_{0}^{T} e^{-\rho t}\left(1+\rho t+\log \left(\psi \pi_{\nu}(t)\right)\right) d t+\psi W_{0}\right] \\
=T e^{-\rho T}-2 \frac{1-e^{-\rho T}}{\rho}+\mathrm{E}\left[\int_{0}^{T} e^{-\rho t}\left(\int_{0}^{t} \tilde{r}(\tau) d \tau\right) d t\right]
\end{array}
$$




$$
\begin{aligned}
& +\min _{\psi>0}\left[\psi W_{0}-\frac{1-e^{-\rho T}}{\rho} \log (\psi)\right] \\
& +\min _{\nu \in \mathcal{N}} \mathrm{E}\left[\int_{0}^{T} e^{-\rho t}\left(\int_{0}^{t}\left(\frac{1}{2}\left|\kappa_{\nu}(\tau)\right|^{2}+\nu_{0}(\tau)\right) d \tau\right) d t\right] .
\end{aligned}
$$

The above implies

$$
\psi^{*}=\frac{1-e^{-\rho T}}{\rho W_{0}}
$$

while the process $\nu^{*}$ identifying the minimax state-price density solves

$$
\nu^{*}(t, \omega)=\arg \min _{\nu \in \mathcal{N}_{t}(\omega)} \frac{1}{2}\left|\kappa_{0}(t, \omega)-\sigma(t, \omega)^{-1}\left(\nu_{-}-\nu_{0} \overline{1}\right)\right|^{2}+\nu_{0}
$$

The optimal consumption, investment and wealth policies are then given by

$$
\begin{gathered}
c_{\nu^{*}}(t)=W_{0} \frac{\rho e^{-\rho t}}{1-e^{-\rho T}} \pi_{\nu^{*}}(t)^{-1}=\frac{\rho}{1-e^{-\rho(T-t)}} W_{\nu^{*}}(t) \\
\theta(t)=\left(\sigma(t) \sigma(t)^{\top}\right)^{-1}\left(\bar{\mu}(t)+\nu_{-}^{*}(t)-\left(\bar{r}(t)+\nu_{0}^{*}(t)\right) \overline{1}\right) W_{\nu^{*}}(t) \\
W_{\nu^{*}}(t)=W_{0} \frac{e^{-\rho t}-e^{-\rho T}}{1-e^{-\rho T}} \pi_{\nu^{*}}(t)^{-1}
\end{gathered}
$$

In particular, the optimal policies for the standard setting with no price dependence can be obtained by taking $\nu^{*}=0$ in the above expressions.

Price pressure: Now consider the price-pressure case discussed in Section 4 , in which $\breve{r} \equiv 0$ and

$$
\breve{\mu}(\alpha, \theta, t, \omega)= \begin{cases}-\frac{A(t, \omega) \theta}{|\theta|} & \text { if } \theta \neq 0 \\ 0 & \text { otherwise }\end{cases}
$$

so that

$$
g(\alpha, \theta, t, \omega)= \begin{cases}-\frac{\theta^{\top} A(t, \omega) \theta}{|\theta|} & \text { if } \theta \neq 0 \\ 0 & \text { otherwise }\end{cases}
$$

and

$$
\mathcal{N}_{t}(\omega)=\left\{\nu \in \mathbb{R}^{n}: \nu_{0}=0 \text { and }\left|A(t, \omega)^{-1} \nu\right| \leq 1\right\} .
$$

We assume that $\sup _{(t, \omega) \in[0, T] \times \Omega}|A(t, \omega)|<\infty$, where $|A|$ denotes the operator norm of the matrix $A$, so that the sets $\mathcal{N}_{t}(\omega)$ are uniformly bounded, since for any $\nu \in \mathcal{N}_{t}(\omega)$

$$
|\nu|=\left|A(t, \omega) A(t, \omega)^{-1} \nu\right| \leq|A(t, \omega)|\left|A(t, \omega)^{-1} \nu\right| \leq \sup _{(t, \omega) \in[0, T] \times \Omega}|A(t, \omega)| .
$$

The solution to $(33)$ is given by $\nu^{*}=\left(0, \nu_{-}^{*}\right)$, where

$$
\nu_{-}^{*}(t)= \begin{cases}\sigma(t) \kappa_{0}(t) & \text { if }\left|A(t)^{-1} \sigma(t) \kappa_{0}(t)\right| \leq 1 \\ {\left[\left(\sigma(t) \sigma(t)^{\top}\right)^{-1}+\lambda(t)\left(A(t) A(t)^{\top}\right)^{-1}\right]^{-1}\left(\sigma(t)^{\top}\right)^{-1} \kappa_{0}(t)} & \text { otherwise }\end{cases}
$$

for some Lagrangian multiplier $\lambda(t)$ such that $\left|A(t)^{-1} \nu^{*}(t)\right|=1$. 
In particular, with a single risky asset $(n=1)$, the above implies

$$
\nu_{-}^{*}(t)= \begin{cases}-(\bar{\mu}(t)-\bar{r}(t)) & \text { if }\left|A(t)^{-1}(\bar{\mu}(t)-\bar{r}(t))\right| \leq 1 \\ -\operatorname{sign}(\bar{\mu}(t)-\bar{r}(t)) A(t) & \text { otherwise }\end{cases}
$$

and

$$
\frac{\theta(t)}{W(t)}= \begin{cases}\sigma(t)^{-2}(\bar{\mu}(t)-\bar{r}(t)+A(t)) & \text { if } \bar{\mu}(t)-\bar{r}(t) \leq-A(t) ; \\ 0 & \text { if }-A(t)<\bar{\mu}(t)-\bar{r}(t)<A(t) ; \\ \sigma(t)^{-2}(\bar{\mu}(t)-\bar{r}(t)-A(t)) & \text { if } \bar{\mu}(t)-\bar{r}(t) \geq A(t) .\end{cases}
$$

This shows that the fraction of wealth invested in the risky asset is always lower than what it would be absent the negative price pressure effect $(A(t)=0)$. However, the marginal propensity to consume is unchanged.

Different borrowing and lending rates: As an additional example, consider the case of different borrowing and lending rates, which corresponds to

$$
\mathcal{N}_{t}(\omega)=\left\{\nu \in \mathbf{R}^{n+1}: 0 \leq \nu_{0} \leq \bar{R}(t, \omega)-\bar{r}(t, \omega) \text { and } \nu_{-}=0\right\} .
$$

In this case, it is easily verified that the solution to (33) is given by $\nu^{*}=\left(\nu_{0}^{*}, 0\right)$, where

$$
\nu_{0}^{*}(t)= \begin{cases}\bar{R}(t)-\bar{r}(t) & \text { if }(\bar{R}(t)-\bar{r}(t))\left|\sigma(t)^{-1} \overline{1}\right|^{2} \leq-\kappa_{0}(t)^{\top} \sigma(t)^{-1} \overline{1}-1 ; \\ -\frac{\kappa_{0}(t)^{\top} \sigma(t)^{-1} \overline{1}+1}{\left|\sigma(t)^{-1} \overline{1}\right|^{2}} & \text { if } 0 \leq-\kappa_{0}(t)^{\top} \sigma(t)^{-1} \overline{1}-1 \leq(\bar{R}(t)-\bar{r}(t))\left|\sigma(t)^{-1} \overline{1}\right|^{2} \\ 0 & \text { otherwise. }\end{cases}
$$

This result first appeared in an Appendix of Cvitanić and Karatzas (1992).

In particular, with a single risky asset the above implies

$$
\nu_{0}^{*}(t)= \begin{cases}\bar{R}(t)-\bar{r}(t) & \text { if } \sigma(t)^{-2}(\mu(t)-\bar{R}(t)) \geq 1 ; \\ \mu(t)-\bar{r}(t)-\sigma(t)^{2} & \text { if } \sigma(t)^{-2}(\mu(t)-\bar{R}(t)) \leq 1 \leq \sigma(t)^{-2}(\mu(t)-\bar{r}(t)) ; \\ 0 & \text { otherwise }\end{cases}
$$

and

$$
\frac{\theta(t)}{W(t)}= \begin{cases}\sigma(t)^{-2}(\mu(t)-\bar{R}(t)) & \text { if } \sigma(t)^{-2}(\mu(t)-\bar{R}(t)) \geq 1 \\ 1 & \text { if } \sigma(t)^{-2}(\mu(t)-\bar{R}(t)) \leq 1 \leq \sigma(t)^{-2}(\mu(t)-\bar{r}(t)) \\ \sigma(t)^{-2}(\mu(t)-\bar{r}(t)) & \text { otherwise. }\end{cases}
$$

As expected, the fraction of wealth invested in the risky asset is always no greater than what it would be without a spread between borrowing and lending rates $(\bar{R}=\bar{r})$. As long as $\mu(t) \leq \bar{r}(t)+\sigma(t)^{2}$, the agent behaves exactly as in the standard setting, investing the fraction $\sigma(t)^{-2}(\mu(t)-\vec{r}(t)) \leq 1$ of his wealth in the stock and lending. However, when $\mu(t)>\bar{r}(t)+\sigma(t)^{2}$ the agent deviates from his optimal policy in the standard setting. In particular, for $\bar{r}(t)+\sigma(t)^{2}<\mu(t) \leq \bar{R}(t)+\sigma(t)^{2}$, the agent keeps all of his wealth invested in the risky asset and neither borrows nor lends. Borrowing only occurs when $\mu(t)>\bar{R}(t)+\sigma(t)^{2}$. As in the previous example, the marginal propensity to consume is unchanged. 


\section{Equilibrium Prices}

We now briefly discuss the partial equilibrium implications of non-price-taking behavior on risk premia. We consider an economy with one non-price-taking agent and a single pricetaking representative agent. Each individual has a time-additive utility function and we denote with $u_{1}(c, t)$ (respectively, $u_{2}(c, t)$ ) the utility function of the non-price-taking agent (respectively, the price-taking-agent) for instantaneous consumption at time $t$. We assume that these functions are three times continuously differentiable in their first argument and continuously differentiable in their second argument. Moreover, the equilibrium prices are Itô processes and a minimax state-price density $\pi_{i}$ exists at the equilibrium prices for all agents $i$ (sufficient conditions for this to happen are given by Theorem 3): in other words,

- letting $c_{i}$ denote the optimal consumption choice for investor $i$, we have

$$
u_{i c}\left(c_{i}(t), t\right)=\psi_{i}(t) \pi_{i}(t)
$$

for $i=1,2$, where

$$
\begin{aligned}
\pi_{1}(t)=\exp ( & -\int_{0}^{t}\left[\sigma(\tau)^{-1}\left(\bar{\mu}(\tau)+\nu_{-}(\tau)-\left(\bar{r}(\tau)-\nu_{0}(\tau)\right) \overline{1}\right)\right]^{\top} d w(\tau) \\
& \left.-\int_{0}^{t}\left(\bar{r}(\tau)+\nu_{0}(\tau)+\frac{1}{2}\left|\sigma(\tau)^{-1}\left(\bar{\mu}(\tau)+\nu_{-}(\tau)-\left(\bar{r}(\tau)-\nu_{0}(\tau)\right) \overline{1}\right)\right|^{2}\right) d \tau\right)
\end{aligned}
$$

for some $\nu \in \mathcal{N}$ and

$$
\begin{aligned}
\pi_{2}(t)=\exp ( & -\int_{0}^{t}\left[\sigma(\tau)^{-1}(\mu(\tau)-\tau(\tau) \overline{1})\right]^{\top} d w(\tau) \\
& \left.-\int_{0}^{t}\left(r(\tau)+\frac{1}{2}\left|\sigma(\tau)^{-1}(\mu(\tau)-r(\tau) \overline{1})\right|^{2}\right) d \tau\right)
\end{aligned}
$$

Let $C=c_{1}+c_{2}$ denote the aggregate consumption process and

$$
\alpha_{i}(t)=-\frac{u_{i c}\left(c_{i}(t), t\right)}{u_{i c c}\left(c_{i}(t), t\right)}
$$

the Arrow-Pratt coefficient of absolute risk tolerance for individual $i$ at time $t$.

We recall that if $X$ and $Y$ are Itô processes with diffusion vectors $\sigma_{X}$ and $\sigma_{Y}$, respectively, their quadratic covariation is the process

$$
[X, Y](t)=\int_{0}^{t} \sigma_{X}(\tau)^{\top} \sigma_{Y}(\tau) d \tau
$$

so that $\frac{d}{d t}[X, Y](t)=\sigma_{X}(t)^{\top} \sigma_{Y}(t)$, which is usually written as $\operatorname{cov}(d X(t), d Y(t))$.

Proposition 1. Under the stated assumptions, the equilibrium risk premia are determined by

$$
\mu(t)-r(t) \overline{1}=\frac{I_{S}(t)^{-1} \operatorname{cov}(d S(t), d C(t))}{\alpha_{1}(t)+\alpha_{2}(t)}+\frac{\alpha_{1}(t) I_{S}(t)^{-1} \operatorname{cov}(d S(t), d Y(t))}{\alpha_{1}(t)+\alpha_{2}(t)},
$$

where $Y(t)=\log \left(u_{1 c}\left(c_{1}(t), t\right) / u_{2 c}\left(c_{2}(t), t\right)\right)$. 
Proof. Let $u_{i c}(t)=u_{i c}\left(c_{i}(t), t\right)$. Under the stated assumptions, $c_{i}$ is an Itô process for $i=1,2$, so that Itô's lemma gives

$u_{i c}(t)=u_{i c}(0)+\int_{0}^{t} u_{i c c}\left(c_{i}(\tau), \tau\right) d c_{i}(\tau)+\int_{0}^{t} u_{i c t}\left(c_{i}(\tau), \tau\right) d \tau+\frac{1}{2} \int_{0}^{t} u_{i c c c}\left(c_{i}(\tau), \tau\right) d\left[c_{i}, c_{i}\right](\tau)$.

Since the quadratic covariation between a continuous semimartingale and a process of finite variation is zero (Jacod and Shiryaev (1987), Proposition I.4.49), we have

$$
\left[u_{i c}, S\right](t)=\left[\int u_{i c c} d c_{i}, S\right](t)=\int_{0}^{t} u_{i c c}\left(c_{i}(\tau), \tau\right) d\left[c_{i}, S\right](\tau),
$$

where the last equality follows from Theorems I.4.40 and I.4.52 in Jacod and Shiryaev (1987). On the other hand, since $u_{i c}=\psi_{i} \pi_{i}$, we also have

$$
\begin{aligned}
{\left[u_{1 c}, S\right](t) } & =\psi_{1}\left[\pi_{1}, S\right](t) \\
& =-\psi_{1} \int_{0}^{t} \pi_{1}(\tau) I_{S}(\tau)\left(\bar{\mu}(\tau)+\nu_{-}(\tau)-\left(\bar{r}(\tau)+\nu_{0}(\tau)\right) \overline{1}\right) d \tau \\
& =-\int_{0}^{t} u_{1 c}(\tau) I_{S}(\tau)\left(\mu(\tau)-\breve{\mu}(\tau)+\nu_{-}(\tau)-\left(r(\tau)-\breve{r}(\tau)+\nu_{0}(\tau)\right) \overline{1}\right) d \tau
\end{aligned}
$$

and

$$
\begin{aligned}
{\left[u_{2 c}, S\right](t) } & =\psi_{2}\left[\pi_{2}, S\right](t) \\
& =-\psi_{2} \int_{0}^{t} \pi_{2}(\tau) I_{S}(\tau)(\mu(\tau)-r(\tau) \overline{1}) d \tau \\
& =-\int_{0}^{t} u_{2 c}(\tau) I_{S}(\tau)(\mu(\tau)-r(\tau) \overline{1}) d \tau
\end{aligned}
$$

The last three equations imply

$$
\alpha_{1}(t)(\mu(t)-r(t) \overline{1})=I_{S}(t)^{-1} \frac{d}{d t}\left[c_{1}, S\right](t)+\alpha_{1}(t)\left(\breve{\mu}(t)-\nu_{-}(t)-\left(\breve{r}(t)-\nu_{0}(t)\right) \overline{1}\right)
$$

and

$$
\alpha_{2}(t)(\mu(t)-r(t) \overline{1})=I_{S}(t)^{-1} \frac{d}{d t}\left[c_{2}, S\right](t)
$$

so that

$$
\begin{aligned}
\left(\alpha_{1}(t)+\alpha_{2}(t)\right)(\mu(t)-r(t) \overline{1}) & =I_{S}(t)^{-1} \frac{d}{d t}[S, C](t)+\alpha_{1}(t)\left(\breve{\mu}(t)-\nu_{-}(t)-\left(\breve{r}(t)-\nu_{0}(t)\right) \overline{1}\right) \\
& =I_{S}(t)^{-1} \frac{d}{d t}[S, C](t)+\alpha_{1}(t) I_{S}(t)^{-1} \frac{d}{d t}[S, Y](t),
\end{aligned}
$$

where the last equality follows from the fact that

$$
Y(t)=\log \left(\frac{u_{1 c}(t)}{u_{2 c}(t)}\right)=\log \left(\frac{\psi_{1} \pi_{1}(t)}{\psi_{2} \pi_{2}(t)}\right)
$$




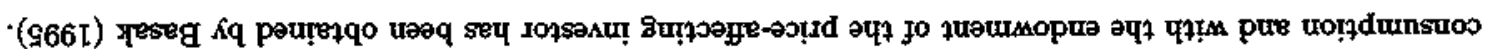

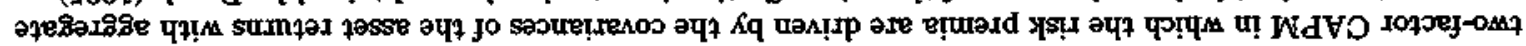

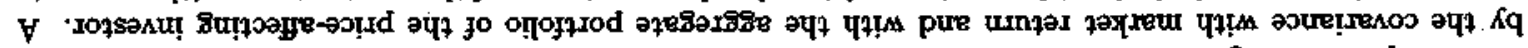

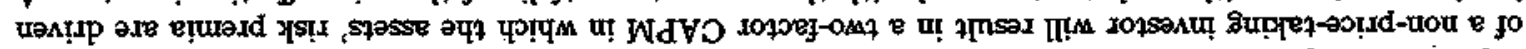

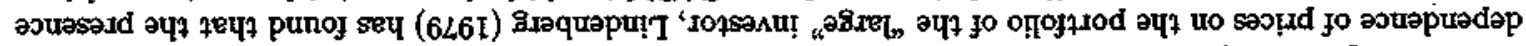

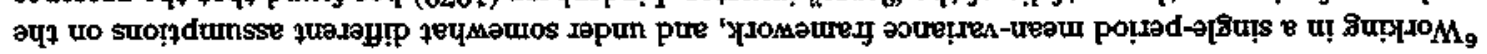

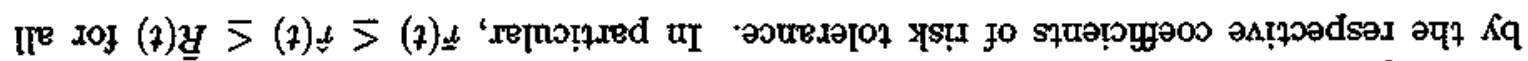
นәл!

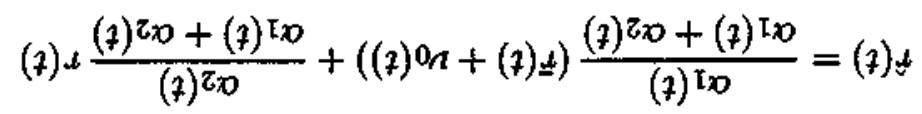

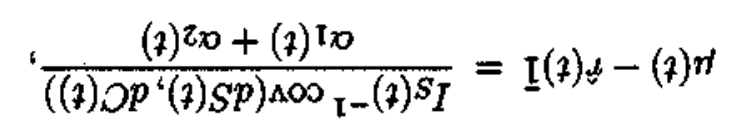

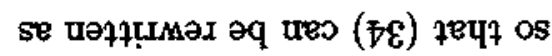$$
\underline{I}((7) 0 \Omega-(7), l)-=(7)[\Lambda ' S] \frac{\not p}{p}{ }_{I}-(7) S_{I}
$$

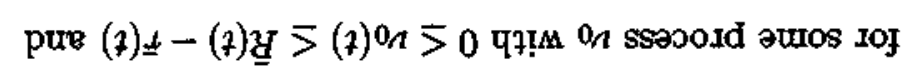

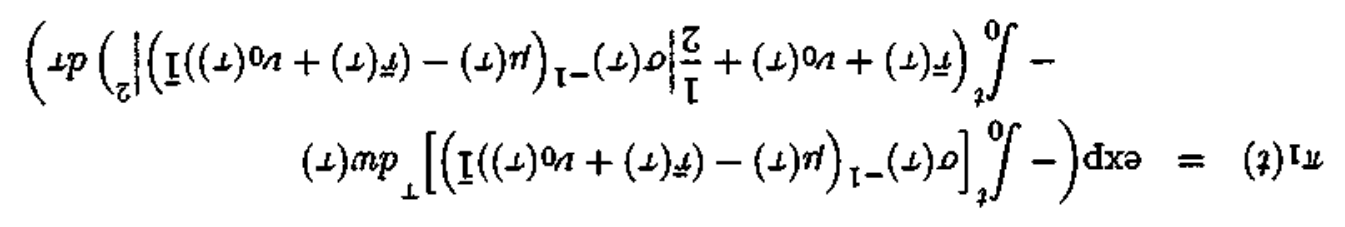

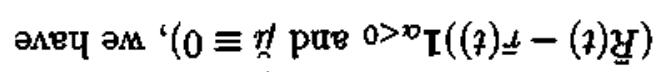

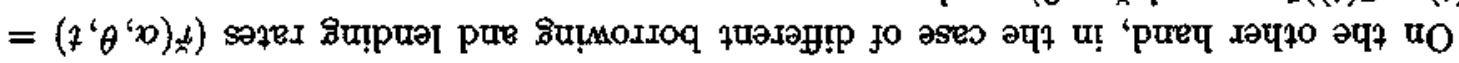

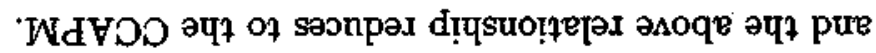

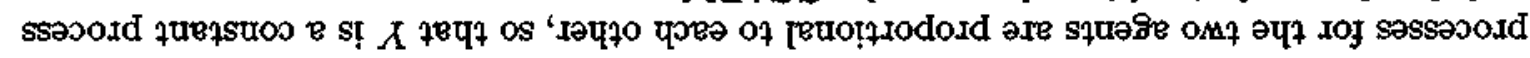

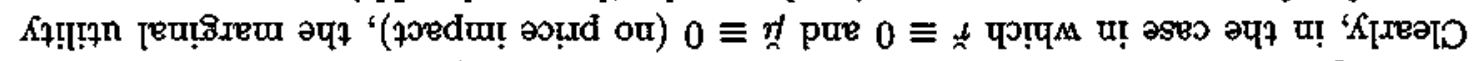

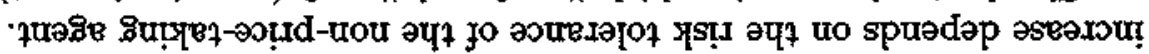

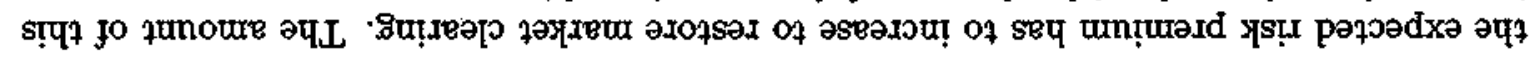

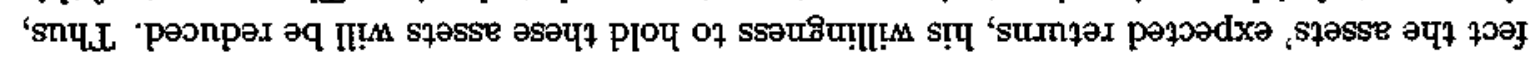

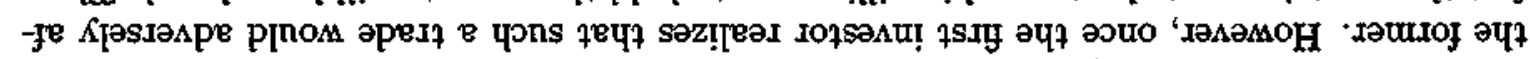

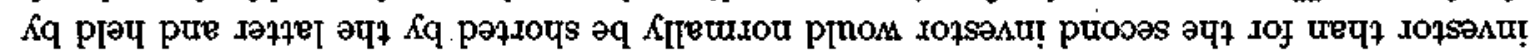

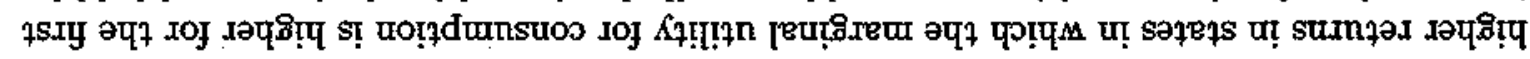

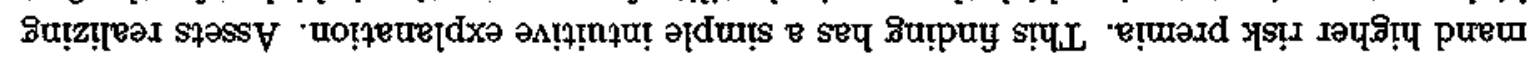

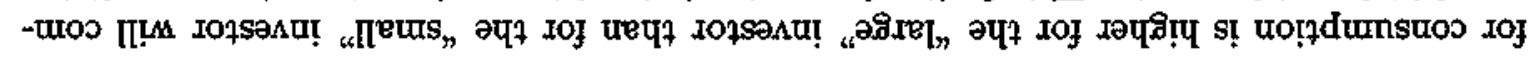

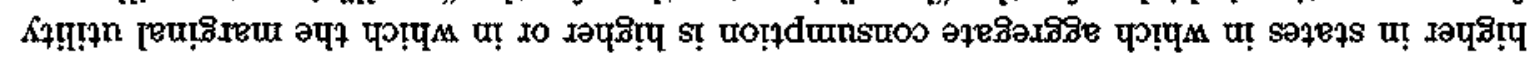

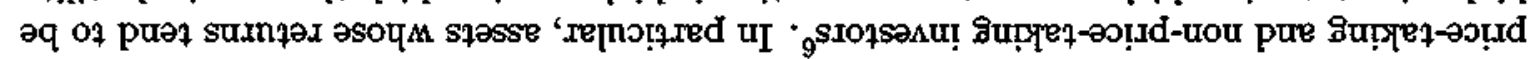

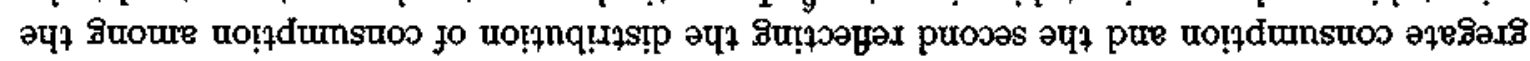

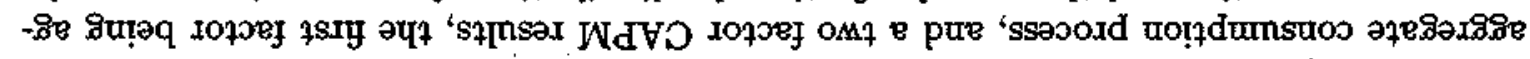

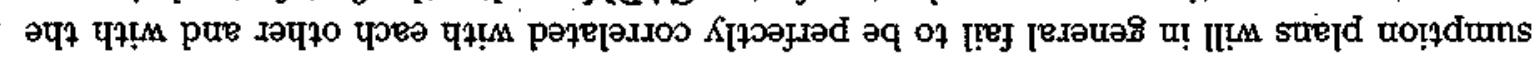

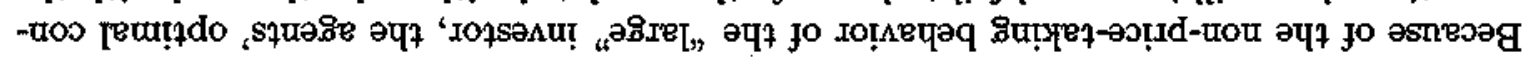

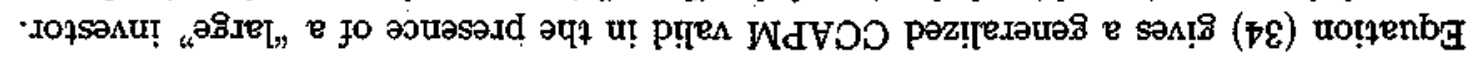


$t \in[0, T]$. This extends to the intertemporal CCAPM a result first obtained by Brennan (1971) and Black (1972) for the static CAPM: with different borrowing and lending rates, the CCAPM still holds, but the expected instantaneous return $\hat{r}$ on a zero-beta portfolio replaces the instantaneous interest rate.

Finally, we remark that in the case of portfolio constraints $((\alpha, \theta) \in K)$ we have

$$
\begin{aligned}
\pi_{1}(t)=\exp ( & -\int_{0}^{t}\left[\sigma(\tau)^{-1}\left(\mu(\tau)+\nu_{-}(\tau)-\left(\bar{r}(\tau)+\nu_{0}(\tau)\right) \overline{1}\right)\right]^{\top} d w(\tau) \\
& \left.-\int_{0}^{t}\left(\bar{r}(\tau)+\nu_{0}(\tau)+\frac{1}{2}\left|\sigma(\tau)^{-1}\left(\mu(\tau)+\nu_{-}(\tau)-\left(\bar{r}(\tau)+\nu_{0}(\tau)\right) \overline{1}\right)\right|^{2}\right) d \tau\right)
\end{aligned}
$$

for some process $\nu$ taking values in the barrier cone of $-K$, and

$$
\left.I_{S}(t)^{-1} \frac{d}{d t}[S, Y](t)=-\left(\nu_{-}(t)-\nu_{0}(t)\right) \overrightarrow{1}\right),
$$

so that (34) can be rewritten as

$$
\mu(t)-r(t) \overline{1}=\frac{I_{S}(t)^{-1} \operatorname{cov}(d S(t), d C(t))}{\alpha_{1}(t)+\alpha_{2}(t)}-\frac{\left.\alpha_{1}(t)\left(\nu_{-}(t)-\nu_{0}(t)\right) \overline{1}\right)}{\alpha_{1}(t)+\alpha_{2}(t)},
$$

which recovers the constrained CCAPM of Cuoco (1995).

\section{Concluding Remarks}

This paper has examined the individual's optimal consumption and investment problem for a "large" investor, whose portfolio choices affect the assets' expected returns. The main result is related to the existence of optimal policies under fairly general assumptions on the security price coefficients and on the income process. As already pointed out, even if we have assumed no bequest function for final wealth, the introduction of such a function can be easily accommodated, and in fact would simplify the statement of some results. Of course, the case in which the agent is maximizing the expected utility from final wealth only could be treated similarly. 


\section{Appendix A}

Proof of Theorem 1: Suppose that $\nu^{*} \in \mathcal{N}$ satisfies (24) and let $W_{\nu^{*}}$ be as in (25). Since the process

$$
M(t)=\pi_{\nu^{*}}(t) W_{\nu^{*}}(t)+\int_{0}^{t} \pi_{\nu^{*}}(\tau)\left(c(\tau)-y(\tau)-\tilde{g}\left(\nu^{*}(\tau), \tau\right)\right) d \tau
$$

is a $P$-martingale with $M(0)=W_{0}$, it follows from the martingale representation theorem that there exists an adapted process $\varphi$ with $\int_{0}^{T}|\varphi(t)|^{2} d t<\infty$ a.s. such that

$$
\pi_{\nu^{*}}(t) W_{\nu^{*}}(t)+\int_{0}^{t} \pi_{\nu^{*}}(\tau)\left(c(\tau)-y(\tau)-\bar{g}\left(\nu^{*}(\tau), \tau\right)\right) d \tau=M(t)=W_{0}+\int_{0}^{t} \varphi(\tau)^{\top} d w(\tau)
$$

Define the portfolio strategy $(\alpha, \theta) \in \Theta$ by

$$
\pi_{\nu^{*}}(t)\left(\sigma(t)^{\top} \theta(t)+W_{\nu^{*}}(t) \kappa_{\nu^{*}}(t)\right)=\varphi(t)
$$

and

$$
\alpha(t)=W_{\nu^{*}}(t)-\sum_{k=1}^{n} \theta_{k}(t)
$$

An application of Itô's lemma then shows that

$$
\begin{aligned}
W_{\nu^{*}}(t)=W_{0} & +\int_{0}^{t}\left(\alpha(\tau)\left(\bar{r}(\tau)+\nu_{0}^{*}(\tau)\right)+\theta(\tau)^{\top}\left(\bar{\mu}(\tau)+\nu_{-}^{*}(\tau)\right)+\tilde{g}\left(\nu^{*}(t), t\right)\right) d \tau \\
& +\int_{0}^{t} \theta(\tau)^{\top} \sigma(\tau) d w(\tau)-\int_{0}^{t}(c(\tau)-y(\tau)) d \tau
\end{aligned}
$$

so that in order to prove that $c$ is feasible we only need to show that

$$
g(\alpha(t), \theta(t), t)=\tilde{g}\left(\nu^{*}(t), t\right)+\alpha(t) \nu_{0}^{*}(t)+\theta(t)^{\top} \nu_{-}^{*}(t), \quad(\lambda \times P) \text {-a.e. }
$$

and that conditions (14)-(15) are satisfied.

Let $\nu$ be the process of (18) and define the process

$$
\begin{aligned}
\zeta(t)= & \int_{0}^{t}\left(\nu_{0}(\tau)-\nu_{0}^{*}(\tau)\right) d \tau \\
& \quad+\int_{0}^{t}\left(\sigma(\tau)^{-1}\left(\nu_{-}(\tau)-\nu_{-}^{*}(\tau)-\left(\nu_{0}(\tau)-\nu_{0}^{*}(\tau)\right) \overline{1}\right)\right)^{\tau}\left(d w(\tau)-\kappa_{\nu^{*}}(\tau) d \tau\right)
\end{aligned}
$$

as well as the sequence of stopping times

$$
\begin{aligned}
\tau_{n}=T \wedge \inf \{t \in & {[0, T]:|\zeta(t)|+\left|\pi_{\nu^{*}}(t)\right|+\left|W_{\nu^{*}}\right| \geq n, } \\
& \text { or } \left.\int_{0}^{t}\left|\sigma(\tau)^{\top} \theta(\tau)+W_{\nu^{*}}(\tau) \kappa_{\nu^{*}}(\tau)\right|^{2} d \tau \geq n\right\} .
\end{aligned}
$$

Then $\tau_{n} \uparrow T$ a.s.. Also, denoting

$$
\nu_{\varepsilon, n}(t)=\nu^{*}(t)+\varepsilon\left[\nu(t)-\nu^{*}(t)\right] 1_{\left\{t \leq \tau_{n}\right\}},
$$


for $\varepsilon \in(0,1)$, we have $\nu_{\varepsilon, n} \in \mathcal{N}$ (because of the convexity of the sets $\mathcal{N}_{t}$ ).

Letting

$$
w_{n}(\varepsilon)=\mathrm{E}\left[\int_{0}^{T} \pi_{\nu_{\varepsilon, n}}(t)\left(c(t)-y(t)-\tilde{g}\left(\nu_{\varepsilon, n}(t), t\right) d t\right]\right.
$$

it can be shown that

$$
\begin{aligned}
& \lim _{\varepsilon \downarrow 0} \frac{w_{n}(0)-w_{n}(\varepsilon)}{\varepsilon} \\
& =\lim _{\varepsilon \downarrow 0} \mathrm{E}\left[\int_{0}^{T} \pi_{\nu^{*}}(t) \frac{c(t)-y(t)-\tilde{g}\left(\nu^{*}(t), t\right)}{\varepsilon}\left(1-\frac{\pi_{\nu_{\varepsilon, n}}(t)}{\pi_{\nu^{*}}(t)}\right) d t\right. \\
& \left.+\int_{0}^{T} \pi_{\nu_{\varepsilon, n}}(t) \frac{\tilde{g}\left(\nu_{\varepsilon, n}(t), t\right)-\tilde{g}\left(\nu^{*}(t), t\right)}{\varepsilon} d t\right] \\
& \leq \lim _{\varepsilon \downarrow 0} \mathrm{E}\left[\int_{0}^{T} \pi_{\nu^{*}}(t) \frac{c(t)-y(t)-\tilde{g}\left(\nu^{*}(t), t\right)}{\varepsilon}\left(1-\frac{\pi_{\nu_{\varepsilon, n}}(t)}{\pi_{\nu^{*}}(t)}\right) d t\right. \\
& \left.+\int_{0}^{\tau_{n}} \pi_{\nu_{\varepsilon, n}}(t)\left(\tilde{g}(\nu(t), t)-\tilde{g}\left(\nu^{*}(t), t\right)\right) d t\right] \\
& =\mathrm{E}\left[\int_{0}^{T} \zeta\left(t \wedge \tau_{n}\right) \pi_{\nu^{*}}(t)\left(c(t)-y(t)-\tilde{g}\left(\nu^{*}(t), t\right)\right) d t\right. \\
& \left.+\int_{0}^{\tau_{n}} \pi_{\nu^{*}}(t)\left(\tilde{g}(\nu(t), t)-\tilde{g}\left(\nu^{*}(t), t\right)\right) d t\right] \\
& =\mathrm{E}\left[\int_{0}^{\tau_{n}} \zeta(t) \pi_{\nu^{*}}(t)\left(c(t)-y(t)-\tilde{g}\left(\nu^{*}(t), t\right)\right) d t+\zeta\left(\tau_{n}\right) \pi_{\nu^{*}}\left(\tau_{n}\right) W_{\nu^{*}}\left(\tau_{n}\right)\right. \\
& \left.+\int_{0}^{\tau_{n}} \pi_{\nu^{*}}(t)\left(\tilde{g}(\nu(t), t)-\tilde{g}\left(\nu^{*}(t), t\right)\right) d t\right]
\end{aligned}
$$

where the first inequality follows from the convexity of $\tilde{g}$, the second equality follows from the dominated convergence theorem, and the third equality follows from the definition of $W_{\nu^{*}}$. On the other hand, by Itô's lemma:

$$
\begin{aligned}
& \zeta\left(\tau_{n}\right) \pi_{\nu^{*}}\left(\tau_{n}\right) W_{\nu^{*}}\left(\tau_{n}\right)=\int_{0}^{\tau_{n}} \zeta(t) \pi_{\nu^{*}}(t)\left(\sigma(t)^{\top} \theta(t)+W_{\nu^{*}}(t) \kappa_{\nu^{*}}(t)\right)^{\top} d w(t) \\
& \quad-\int_{0}^{\tau_{n}} \zeta(t) \pi_{\nu^{*}}(t)\left(c(t)-y(t)-\tilde{g}\left(\nu^{*}(t), t\right)\right) d t+\int_{0}^{\tau_{n}} \pi_{\nu^{*}}(t) W_{\nu^{*}}(t)\left(\nu_{0}(t)-\nu_{0}^{*}(t)\right) d t \\
& \quad+\int_{0}^{\tau_{n}} \pi_{\nu^{*}}(t) W_{\nu^{*}}(t)\left(\sigma(t)^{-1}\left(\nu_{-}(t)-\nu_{-}^{*}(t)-\left(\nu_{0}(t)-\nu_{0}^{*}(t)\right) \overline{1}\right)\right)^{\top}\left(d w(\tau)-\kappa_{\nu^{*}}(\tau) d \tau\right) \\
& \quad+\int_{0}^{\tau_{n}} \pi_{\nu^{*}}(t)\left(\sigma(t)^{-1}\left(\nu_{-}(t)-\nu_{-}^{*}(t)-\left(\nu_{0}(t)-\nu_{0}^{*}(t)\right) \overline{1}\right)\right)^{\top}\left(\sigma(t)^{\top} \theta(t)+W_{\nu^{*}}(t) \kappa_{\nu^{*}}(t)\right) d t .
\end{aligned}
$$

Taking expectations and using the fact that the stochastic integrals in the above expression are martingales shows that

$$
\begin{aligned}
& \mathrm{E}\left[\int_{0}^{\tau_{n}} \zeta(t) \pi_{\nu^{*}}(t)\left(c(t)-y(t)-\tilde{g}\left(\nu^{*}(t), t\right)\right) d t+\zeta\left(\tau_{n}\right) \pi_{\nu^{*}}\left(\tau_{n}\right) W_{\nu^{*}}\left(\tau_{n}\right)\right] \\
& \quad=\mathrm{E}\left[\int_{0}^{\tau_{n}} \pi_{\nu^{*}}(t)\left(\alpha(t)\left(\nu_{0}(t)-\nu_{0}^{*}(t)\right)+\theta(t)^{\top}\left(\nu_{-}(t)-\nu_{-}^{*}(t)\right)\right) d t\right]
\end{aligned}
$$


Substituting the last equality in (38) and recalling the definition of $\nu$ gives

$$
\begin{aligned}
& \lim _{\varepsilon l 0} \frac{w_{n}(0)-w_{n}(\varepsilon)}{\varepsilon} \\
& \quad=\mathrm{E}\left[\int_{0}^{\tau_{n}} \pi_{\nu^{*}}(t)\left(\alpha(t)\left(\nu_{0}(t)-\nu_{0}^{*}(t)\right)+\theta(t)^{\top}\left(\nu_{-}(t)-\nu_{-}^{*}(t)\right)+\tilde{g}(\nu(t), t)-\bar{g}\left(\nu^{*}(t), t\right)\right) d t\right] \\
& \quad=\mathrm{E}\left[\int_{0}^{\tau_{n}} \pi_{\nu^{*}}(t)\left(g(\alpha(t), \theta(t), t)-\alpha(t) \nu_{0}^{*}(t)-\theta(t)^{\top} \nu_{-}^{*}(t)-\tilde{g}\left(\nu^{*}(t), t\right)\right) d t\right] .
\end{aligned}
$$

By (24), the left-hand side of the last expression is nonnegative. Since

$$
\tilde{g}\left(\nu^{*}(t), t\right) \geq g(\alpha(t), \theta(t), t)-\alpha(t) \nu_{0}^{*}(t)-\theta(t)^{\top} \nu_{-}^{*}(t)
$$

by the definition of $\tilde{g}$, we conclude that (37) must hold.

Finally, we turn to conditions (14)-(15). Clearly, $W_{\nu^{m}}(T)=0$, and hence (15) is satisfied. Also, we have (from (3), the boundedness of $\nu^{*}$ and the martingale property of $\xi_{\nu^{*}}$ )

$$
W_{\nu^{*}}(t) \geq-K\left(\beta_{0}(t) \xi_{\nu^{*}}(t)\right)^{-1} \mathrm{E}\left[\int_{t}^{T} \xi_{\nu^{*}}(\tau) y(\tau) d \tau \mid \mathcal{F}_{t}\right] \geq-K T \bar{y} \beta_{0}(t)^{-1},
$$

for some $K>0$, where $\bar{y}<\infty$ is an upper bound on $y$, so that (14) is also satisfied.

ProOF of THEOREM 2: Define the consumption policy $c^{*}=c_{\nu^{*}}$ and the wealth process $W^{*}=W_{\nu^{*}}$ by (31) and (25), respectively (the latter is finite because of (30) and Assumption 5). In order to prove that $c^{*}$ is optimal we will proceed in three steps: first we will show that $c^{*} \in \mathcal{C}_{+}^{*}$, then that $U\left(c^{*}\right) \geq U(c)$ holds for all feasible consumption processes $c \in \mathcal{C}_{+}^{*}$, and finally that $c^{*}$ is feasible.

Step 1: By the continuity of $f$ and $\pi_{\nu^{*}}$, it is clear that $\int_{0}^{T} c^{*}(t) d t<\infty$ holds a.s.. Also, from (29) we have

$$
\mathrm{E}\left[\int_{0}^{T} u\left(c^{*}(t), t\right)^{-} d t\right] \leq \int_{0}^{T} u(1, t)^{-} d t+\psi^{*} \mathrm{E}\left[\int_{0}^{T} \pi_{\nu^{*}}(t) d t\right]<\infty
$$

Hence, $c^{*} \in \mathcal{C}_{+}^{*}$.

Step 2: By (12), (30) implies that

$$
\mathrm{E}\left[\int_{0}^{T} \pi_{\nu^{*}}(t) f\left(\psi \pi_{\nu^{*}}(t), t\right) d t\right]<\infty
$$

holds for all $\psi \in(0, \infty)$. By the optimality of $\psi^{*}$, we then have

$$
\begin{aligned}
0= & \lim _{\varepsilon \rightarrow 0} \frac{J\left(\psi^{*}+\varepsilon, \nu^{*}\right)-J\left(\psi^{*}, \nu^{*}\right)}{\varepsilon} \\
= & \mathrm{E}\left[\int_{0}^{T} \lim _{\varepsilon \rightarrow 0} \frac{\tilde{u}\left(\left(\psi^{*}+\varepsilon\right) \pi_{\nu^{*}}(t), t\right)-\tilde{u}\left(\psi^{*} \pi_{\nu^{*}}(t), t\right)}{\varepsilon} d t\right. \\
& \quad+\int_{0}^{T} \pi_{\nu^{*}}(t)\left(y(t)+\tilde{g}\left(\nu^{*}(t), t\right) d t+W_{0}\right] \\
= & W_{0}-\mathrm{E}\left[\int_{0}^{T} \pi_{\nu^{*}}(t)\left(c^{*}(t)-y(t)-\tilde{g}\left(\nu^{*}(t), t\right)\right) d t\right]
\end{aligned}
$$


where the second equality follows from Lebesgue's dominated convergence theorem and (39), using the fact that

$$
\begin{gathered}
\left|\frac{\tilde{u}\left(\left(\psi^{*}+\varepsilon\right) \pi_{\nu^{*}}(t), t\right)-\tilde{u}\left(\psi^{*} \pi_{\nu^{*}}(t), t\right)}{\varepsilon}\right| \leq \frac{\tilde{u}\left(\left(\psi^{*}-|\varepsilon|\right) \pi_{\nu^{*}}(t), t\right)-\tilde{u}\left(\psi^{*} \pi_{\nu^{*}}(t), t\right)}{|\varepsilon|} \\
\leq \pi_{\nu^{*}}(t) f\left(\left(\psi^{*}-|\varepsilon|\right) \pi_{\nu^{*}}(t), t\right) \leq \pi_{\nu^{*}}(t) f\left(\left(\psi^{*} / 2\right) \pi_{\nu^{*}}(t), t\right)
\end{gathered}
$$

for $|\varepsilon|<\psi^{*} / 2$, because $\tilde{u}(\cdot, t)$ is decreasing and convex, $\frac{\partial}{\partial y} \tilde{u}(y, t)=-f(y, t)$, and $f(\cdot, t)$ is decreasing. Therefore

$$
\mathrm{E}\left[\int_{0}^{T} \pi_{\nu^{*}}(t)\left(c^{*}(t)-y(t)-\tilde{g}\left(\nu^{*}(t), t\right)\right) d t\right]=W_{0} .
$$

Next, let $c \in \mathcal{C}_{+}^{*}$ be any feasible consumption process. Since by concavity

$$
u(f(y, t), t)-u(c, t) \geq y[f(y, t)-c] \quad \forall c>0, y>0,
$$

we have from (21)

$$
U\left(c^{*}\right)-U(c)=\mathrm{E}\left[\int_{0}^{T}\left(u\left(c^{*}(t), t\right)-u(c(t), t)\right) d t\right] \geq \psi^{*} \mathrm{E}\left[\int_{0}^{T} \pi_{\nu^{*}}(t)\left(c^{*}(t)-c(t)\right) d t\right] \geq 0 .
$$

Hence, $c^{*}$ must be optimal provided it is feasible.

Step 3: By the martingale representation theorem, there exists an adapted process $\varphi$ with $\int_{0}^{T}|\varphi(t)|^{2} d t<\infty$ a.s. such that

$$
\pi_{\nu^{*}}(t) W^{*}(t)+\int_{0}^{t} \pi_{\nu^{*}}(\tau)\left(c^{*}(\tau)-y(\tau)-\tilde{g}\left(\nu^{*}(\tau), \tau\right)\right) d \tau=W_{0}+\int_{0}^{t} \varphi(\tau)^{\top} d w(\tau)
$$

Defining the portfolio strategy $\theta$ by (36), it can be verified as in the proof of Theorem 1 that (14) and (15) are satisfied, and hence in order to prove that $c^{*}$ is feasible we are only left to show that (13) holds. As in the proof of Theorem 1, this is equivalent to showing that (37) is satisfied.

Let $\nu \in \mathcal{N}$ be the process of (18) and define the process $\zeta$, as well as the stopping times $\tau_{n}$, as in the proof of Theorem 1. For $\varepsilon \in(0,1)$, let

$$
\nu_{\varepsilon, n}(t)=\nu^{*}(t)+\varepsilon\left[\nu(t)-\nu^{*}(t)\right] 1_{\left\{t \leq \tau_{n}\right\}} .
$$

Using Lebesgue's dominated convergence theorem and Itô's lemma as in the proof of Theorem 1 , it can be verified that

$$
\begin{aligned}
& \lim _{\varepsilon \downarrow 0} \frac{J\left(\psi^{*}, \nu_{\varepsilon, n}\right)-J\left(\psi^{*}, \nu^{*}\right)}{\varepsilon} \\
&=\lim _{\varepsilon \downarrow 0} \mathrm{E}\left[\int_{0}^{T} \frac{\tilde{u}\left(\psi^{*} \nu_{\varepsilon, n}(t), t\right)-\tilde{u}\left(\psi^{*} \pi_{\nu^{*}}(t), t\right)}{\varepsilon} d t\right. \\
& \quad-\psi^{*} \int_{0}^{T} \pi_{\nu^{*}}(t) \frac{y(t)+\tilde{g}\left(\nu^{*}(t), t\right)}{\varepsilon}\left(1-\frac{\pi_{\nu_{\varepsilon, n}}(t)}{\pi_{\nu^{*}}(t)}\right) d t
\end{aligned}
$$




$$
\begin{gathered}
\left.+\psi^{*} \int_{0}^{T} \pi_{\nu_{\varepsilon, n}}(t) \frac{\tilde{g}\left(\nu_{\varepsilon, n}(t), t\right)-\tilde{g}\left(\nu^{*}(t), t\right)}{\varepsilon} d t\right] \\
\leq \psi^{*} \mathrm{E}\left[\int_{0}^{T} \zeta\left(t \wedge \tau_{n}\right) \pi_{\nu^{*}}(t)\left(c^{*}(t)-y(t)-\dot{g}\left(\nu^{*}(t), t\right)\right) d t\right. \\
\left.\quad+\int_{0}^{\tau_{n}} \pi_{\nu^{*}(t)}\left(\tilde{g}(\nu(t), t)-\bar{g}\left(\nu^{*}(t), t\right)\right) d t\right] \\
=\psi^{*} \mathrm{E}\left[\int_{0}^{\tau_{n}} \pi_{\nu^{*}(t)}\left(g(\alpha(t), \theta(t), t)-\alpha(t) \nu_{0}^{*}(t)-\theta(t)^{\top} \nu_{-}^{*}(t)-\tilde{g}\left(\nu^{*}(t), t\right)\right) d t\right] .
\end{gathered}
$$

Since $J\left(\psi^{*}, \nu_{\varepsilon, n}\right)$ reaches a minimum at $\varepsilon=0$, the last term is nonnegative. Condition (37) then follows by the same argument used in the proof of Theorem 1. 


\section{Appendix B}

This Appendix is devoted to the proof of Theorem 3.

Assume for simplicity that $\bar{r}(t)=0$ and that $\sigma(t)$ is the identity matrix, for all $t \in[0, T]$. Also, assume that $\bar{\mu} \equiv 0$ (alternatively, one can work under the risk-neutral measure $Q_{0}$ instead of the original measure $P$ ). Since the dual functional $J(\psi, \nu)$ is not convex in $\nu$, we start by reformulating the dual problem $\left(P^{*}\right)$ as a minimization problem over a closed and convex subset of a $L^{2}$ space.

Let $\mathcal{M}$ denote the progressive $\sigma$-field (i.e., the smallest $\sigma$-field with respect to which all progressive processes are measurable), and let $L_{n}^{2}=L_{n}^{2}([0, T] \times \Omega, \mathcal{M}, \lambda \times P)$ denote the space of all progressively measurable $n$-dimensional processes $X$ with

$$
\mathrm{E}\left[\int_{0}^{T}|X(t)|^{2} d t\right]<\infty
$$

By the boundedness of $\mathcal{N}$, it is easily seen that the set $\Pi=\left\{\pi_{\nu}: \nu \in \mathcal{N}\right\}$ is uniformly bounded in $L_{1}^{2}$. Because of this square-integrability, the positivity of each $\pi_{\nu} \in \Pi$, and the uniqueness of the semimartingale representation

$$
\pi_{\nu}(t)=1+\int_{0}^{t} \pi_{\nu}(\tau) \nu_{0}(\tau) d \tau+\int_{0}^{t} \pi_{\nu}(\tau) \nu_{-}(\tau)^{\top} d w(\tau)
$$

$\nu$ is uniquely determined by $\pi_{\nu}$ (up to $(\lambda \times P)$-equivalence). The dual problem can therefore be regarded as a problem in $\left(\psi, \pi_{\nu}\right)$, and rewritten as

$$
\inf _{\left(\psi, \pi_{\nu}\right) \in(0, \infty) \times \Pi} \bar{J}\left(\psi, \pi_{\nu}\right)
$$

where

$$
\tilde{J}\left(\psi, \pi_{\nu}\right)=\mathrm{E}\left[\int_{0}^{T} \tilde{u}\left(\psi \pi_{\nu}(t), t\right) d t+\psi \int_{0}^{T} \pi_{\nu}(t)(y(t)+\tilde{g}(\nu(t), t)) d t+\psi W_{0}\right] .
$$

It is easy to see that, under the assumptions of Theorem 3 , a sufficient condition for the minimum in $\left(P^{* *}\right)$ to be attained is that for all $\psi \in(0, \infty)$ there exists a solution to the problem

$$
\inf _{\pi \in \Pi} \tilde{J}(\psi, \pi) \text {. }
$$

In fact, letting $V(\psi)$ denote the value function in (43), it can be verified that $V$ is strictly convex and continuous on $(0, \infty)$, and that it satisfies the coercitivity conditions $V(0+)=$ $V(\infty)=\infty$. Therefore, $V$ must attain a (unique) minimum on $(0, \infty)$, and hence $\left(P^{* *}\right)$ has a solution.

By Proposition 2.1.2 in Ekeland and Temam (1976), in order to prove that the infimum in (43) is attained it is sufficient to show that $(i) \Pi$ is convex and closed in $L_{1}^{2}$, and $(i i) \tilde{J}(\psi, \cdot)$ is convex and lower semicontinuous on $\Pi$. Indeed, since any minimizing sequence $\left\{\pi_{n}\right\}$ is bounded in $L_{1}^{2}$, one can extract a (minimizing) subsequence, converging weakly to some $\pi^{*} \in \Pi$ (since closedness is preserved under weak convergence because of convexity). By 
the lower semicontinuity of $\tilde{J}(\psi, \cdot)$ (which is also preserved under weak convergence because of convexity), we get

$$
\tilde{J}\left(\psi, \pi^{*}\right) \leq \lim _{n \neq \infty} \tilde{J}\left(\psi, \pi_{n}\right)=\inf _{\pi \in \Pi} \tilde{J}(\psi, \pi)
$$

and $\pi^{*}$ is optimal.

We will prove $(i)$ and $(i i)$ through three lemmas. The next lemma implies in particular that $\Pi$ is convex.

Lemma B1. The set

$$
\hat{\Pi}=\left\{\left(\pi_{\nu}, \pi_{\nu} \nu\right): \nu \in \mathcal{N}\right\}
$$

is convex and closed in $L_{n+2}^{2}$.

Proof. Convexity of $\hat{\Pi}$ follows immediately from (42).

Next, suppose that $\left\{\left(\pi_{\nu_{n}}, \pi_{\nu_{n}} \nu_{n}\right)\right\} \subset \hat{\Pi}$ and $\left\{\left(\pi_{\nu_{n}}, \pi_{\nu_{n}} \nu_{n}\right)\right\}$ converges to $(\pi, \rho)$ in $L_{n+2}^{2}$. We will start by showing that $\pi>0$ a.e., so that we can define the processes $\nu$ by $\nu=\rho / \pi$. Indeed, assume that is not the case, so that there is a set $A$ of positive $\lambda \times P$ measure on which $\pi=0$. Then, since

$$
\pi_{\nu_{n}}(t)=\exp \left(\int_{0}^{t} \nu_{n-}(\tau)^{\top} d w(\tau)-\int_{0}^{t}\left(\nu_{n 0}(\tau)-\frac{1}{2}\left|\nu_{n-}(\tau)\right|^{2}\right) d \tau\right)
$$

it follows there is a (relabeled) subsequence along which the processes

$$
I_{n}(t)=\int_{0}^{t} \nu_{n-}(\tau)^{\top} d w(\tau)
$$

converge to $-\infty$ a.e. on $A$. Therefore, letting

$$
B_{n}^{N}=\left\{(t, \omega): I_{n}(t)>-N\right\}
$$

and denoting by $\bar{B}$ the complement of a set $B$, we get (mimicking an argument by Beneš (1971))

$$
\begin{aligned}
(\lambda \times P)(A) & =\mathrm{E}\left[\int_{0}^{T} 1_{A}(t) d t\right]=\mathrm{E}\left[\int_{0}^{T}\left(1_{A \cap B_{n}^{N}}(t)+1_{A \cap B_{n}^{N}}(t)\right) d t\right] \\
& \leq \mathrm{E}\left[\int_{0}^{T} 1_{A \cap B_{n}^{N}}(t) d t\right]+\frac{1}{N^{2}} \mathrm{E}\left[\int_{0}^{T} I_{n}(t)^{2} d t\right] \\
& \leq \varepsilon+\frac{1}{N^{2}} \mathrm{E}\left[\int_{0}^{T}\left(\int_{0}^{t}\left|\nu_{n-1}\right|^{2}(\tau) d \tau\right) d t\right]
\end{aligned}
$$

for arbitrary $\varepsilon>0$ and $N>0$ and $n$ large enough. Letting $N \rightarrow \infty$ and recalling that the set $\left\{\nu_{n}\right\}$ is uniformly bounded we get the contradiction.

Since $\pi_{\nu_{n}} \nu_{n} \rightarrow \pi \nu$ in $L_{n+1}^{2}$, the processes $\int_{0}^{t} \pi_{\nu_{n}}(\tau) \nu_{n 0}(\tau) d \tau$ and $\int_{0}^{t} \pi_{\nu_{n}}(\tau) \nu_{n-}(\tau)^{\top} d w(\tau)$ converge to $\int_{0}^{t} \pi(\tau) \nu_{0}(\tau) d \tau$ and $\int_{0}^{t} \pi(\tau) \nu_{-}(\tau)^{\top} d w(\tau)$, respectively, in $L_{1}^{2}$. By the semimartingale representation (42) for $\pi_{\nu_{n}}$, this implies that $\pi_{\nu_{n}}$ converges to $\pi_{\nu}$ in $L_{1}^{2}$ and hence that $\pi=\pi_{\nu}$.

Finally, we prove that $\nu \in \mathcal{N}$. Since $\left\{\left(\pi_{\nu_{n}}, \pi_{\nu_{n}} \nu_{n}\right)\right\}$ converges to $(\pi, \pi \nu)$ in $L_{n+2}^{2}$, we can assume (by passing to a subsequence) that convergence also occurs a.e.. This implies that $\nu_{n}$ converges to $\nu$ a.e., and hence $\nu \in \mathcal{N}$ (since $\mathcal{N}_{t}(\omega)$ is closed for all $(t, \omega) \in[0, T] \times \Omega$ ). 
In the following we denote by $\operatorname{co}(A)$ the convex hull of a set $A$ and by $\overline{c o}(A)$ its closed convex hull (see, e.g., Dunford and Schwartz (1988), Definition V.2.2). The next lemma implies in particular that $\Pi$ is closed.

Lemma B2. Suppose that $\left\{\pi_{\nu_{n}}\right\} \subset \Pi$ converges to $\pi$ in $L_{1}^{2}$. Then $\pi=\pi_{\nu}$ for some $\nu \in \mathcal{N}$ and

$$
\left(\pi_{\nu}, \pi_{\nu} \nu\right) \in \overline{\mathrm{co}}\left(\left\{\left(\pi_{\nu_{n}}, \pi_{\nu_{n}} \nu_{n}\right)\right\}\right) .
$$

Proof. Suppose that $\left\{\pi_{\nu_{n}}\right\} \subset \Pi$ converges to $\pi$ in $L_{1}^{2}$. The same argument used at the beginning of the proof of Lemma B1 then implies that $\pi>0$. Since the set $\left\{\left(\pi_{\nu}, \pi_{\nu} \nu\right)\right.$ : $\nu \in \mathcal{N}\}$ is uniformly bounded in $L_{n+2}^{2}$, it is weakly sequentially compact (Dunford and Schwartz (1988), Theorem II.3.28), and we can then assume (by possibly passing to a subsequence) that there exists a process $\nu$ such that $\left\{\left(\pi_{\nu_{n}}, \pi_{\nu_{n}} \nu_{n}\right)\right\}$ converges to $\{(\pi, \pi \nu)\}$ weakly in $L_{n+2}^{2}$. By Mazur's Lemma (Dunford and Schwartz (1988), Corollary V.3.14), $\{(\pi, \pi \nu)\} \in \overline{c o}\left(\left\{\left(\pi_{\nu_{n}}, \pi_{\nu_{n}} \nu_{n}\right)\right\}\right)$, and hence $(\pi, \pi \nu)$ belongs to the set $\hat{\Pi}$ of (44) by Lemma B1. Therefore, $\pi=\pi_{\nu}$.

Lemma B3. The functional $\tilde{J}(\psi, \cdot)$ is convex and lower semicontinuous on $\Pi$.

Proof. It follows immediately from the convexity of the set $\hat{\Pi}$ in (44), that

$$
\alpha \pi_{\nu_{1}}+(1-\alpha) \pi_{\nu_{2}}=\pi_{\nu}
$$

where

$$
\nu=\frac{\alpha \pi_{\nu_{1}}}{\pi_{\nu}} \nu_{1}+\frac{(1-\alpha) \pi_{\nu_{2}}}{\pi_{\nu}} \nu_{2}
$$

By the convexity of $\tilde{g}$, this implies

$$
\pi_{\nu}(t) \tilde{g}(\nu(t), t) \leq \alpha \pi_{\nu_{1}}(t) \tilde{g}\left(\nu_{1}(t), t\right)+(1-\alpha) \pi_{\nu_{2}}(t) \tilde{g}\left(\nu_{2}(t), t\right) .
$$

Together with convexity of $\tilde{u}$, this implies the convexity of $\tilde{J}(\psi, \cdot)$.

Next, we claim that $\tilde{J}(\psi, \cdot)$ is lower semicontinuous on $\Pi$. In fact, suppose that this is not the case. Then there is a $\alpha>0$, a $\pi=\pi_{\nu} \in \Pi$ and a sequence $\left\{\pi_{\nu_{n}}\right\}$ converging to $\pi$ in $L_{1}^{2}$ such that

$$
J\left(\psi, \pi_{\nu_{n}}\right) \leq \alpha<J(\psi, \pi) \quad \text { for all } n .
$$

By Lemma B2, we can then find a sequence $\left\{\pi_{\hat{\nu}_{n}}\right\} \subset \operatorname{co}\left(\left\{\pi_{\nu_{n}}\right\}\right)$ such that $\left(\pi_{\hat{\nu}_{n}}, \hat{\nu}_{n}\right)$ converges to $(\pi, \nu)$ a.e., and it follows from the convexity of $\tilde{J}(\psi, \cdot)$ that $\tilde{J}\left(\psi, \pi_{\hat{\nu}_{n}}\right) \leq \alpha$ for all $n$. On the other hand, since $\tilde{u}(y, t)$ is convex in its first argument and decreasing in its second argument, there exist constants $a, b$ such that $\tilde{u}(y, t) \geq \tilde{u}(y, T) \geq-(a+b y)$ for $y>0$. We then have from Fatou's lemma and the uniform integrability of $\left\{\pi_{\hat{\nu}_{n}}\right\}$ that

$$
\alpha<\tilde{J}(\psi, \pi) \leq \liminf _{n \uparrow \infty} \tilde{J}\left(\psi, \pi_{\hat{\nu}_{n}}\right) \leq \alpha,
$$

where we have used the continuity of $\tilde{u}$ and the lower semicontinuity of $\tilde{g}$. The contradiction establishes the lower semicontinuity of $\tilde{J}(\psi, \cdot)$. 
The proof of the existence of a solution to the dual problem ( $P^{*}$ ) is now complete Finally, to show that (30) is satisfied, note that condition (d) of the Theorem implies

$$
y f(y, t) \leq a+(1-b) u(f(y, t), t)
$$

and hence

$$
\mathrm{E}\left[\int_{0}^{T} \pi_{\nu^{*}}(t) f\left(\psi^{*} \pi_{\nu^{*}}(t)\right) d t\right] \leq \frac{a}{b \psi^{*}}+\frac{1-b}{b \psi^{*}} \mathrm{E}\left[\int_{0}^{T} \tilde{u}\left(\psi^{*} \pi_{\nu^{*}}(t), t\right) d t\right]<\infty
$$

where the last inequality follows from the fact that $\left|J\left(\psi^{*}, \nu^{*}\right)\right|<\infty$ and $y / B$ is bounded. 


\section{References}

BasaK, S. (1995), "Dynamic Consumption and Portfolio Choice and Asset Pricing with Non-Price-Taking Agents", mimeo, The Wharton School, University of Pennsylvania.

BENEŠ, V.E. (1971), "Existence of Optimal Stochastic Control Laws", SIAM Journal of Control 8, 446-475.

BLACK, F. (1972), "Capital Market Equilibrium with Restricted Borrowing", Journal of Business, 45, 444-455.

BRENNAN, M.J. (1971), "Capital Market Equilibrium with Divergent Borrowing and Lending Rates", Journal of Financial and Quantitative Analysis, 6, 1197-1205.

Chow, Y.S. AND H. Teicher (1988), Probability Theory, Springer-Verlag, New York.

Cox, J.C., ANd C.-F. HuAng (1989), "Optimal Consumption and Portfolio Policies when Asset Prices Follow a Diffusion Process", Journal of Economic Theory 49, 33-83.

Cox, J.C., AND C.-F. HUANG (1991), "A Variational Problem Arising in Financial Economics", Journal of Mathematical Economics 20, 465-487.

Cuoco, D. (1995), "Optimal Consumption and Equilibrium Prices with Portfolio Constraints and Stochastic Income", forthcoming in Journal of Economic Theory.

Cvitanić, J. (1995), "Nonlinear Financial Markets: Hedging and Portfolio Optimization", mimeo, Department of Statistics, Columbia University.

Cvitanić, J. and I. Karatzas (1992), "Convex Duality in Constrained Portfolio Optimization", Annals of Applied Probability 2, 767-818.

Cvitanić, J. AND I. Karatzas (1993), "Hedging Contingent Claims with Constrained Portfolios ", Annals of Applied Probability 3, 652-681.

Cvitanić, J. AND J. MA (1994), "Hedging Options for a Large Investor and ForwardBackward SDE's", mimeo, Department of Statistics, Columbia University.

DuffiE, D. AND L.G. EPSTEIN (1992), "Stochastic Differential Utility", Econometrica 60, 353-394.

Dybvig, P.H. AND C.-F. HuANG (1989), "Nonnegative Wealth, Absence of Arbitrage, and Feasible Consumption Plans", Review of Financial Studies 1, 377-401.

Ekeland, I., AND R. TemaM (1976), Convex Analysis and Variational Problems, North Holland, Amsterdam.

El Karoui, N., S. Peng, And M.C. Quenez (1995), "Backwards Stochastic Differential Equations in Finance and Optimization", forthcoming in Mathematical Finance.

HE, H. AND H.F. PAGÈs (1993), "Labor Income, Borrowing Constraints, and Equilibrium Asset Prices: A Duality Approach", Economic Theory 3, 663-696.

He, H. AND N.D. Pearson (1991), "Consumption and Portfolio Policies with Incomplete Markets and Short-Sale Constraints: The Infinite Dimensional Case", Journal of Economic Theory 54, 259-304. 
JACOD, J. AND A.N. ShIRYAEV (1987), Limit Theorems for Stochastic Processes, SpringerVerlag, New York.

JARrow, R.A. (1992), "Market Manipulation, Bubbles, Corners, and Short Squeezes", · Journal of Financial and Quantitative Analysis 27, 311-336.

JARROW, R.A. (1994), "Derivative Security Markets, Market Manipulation, and Option Pricing Theory", Journal of Financial and Quantitative Analysis 29, 241-261.

Karatzas, I., J.P. Lehoczky, AND S.E. Shreve (1987), "Optimal Portfolio and Consumption Decisions for a 'Small Investor' on a Finite Horizon", SIAM Journal of Control and Optimization 25, 1557-1586.

Karatzas, I., J.P. LehoczKY, AND S.E. Shreve (1990), "Existence and Uniqueness of Multi-Agent Equilibrium in a Stochastic, Dynamic Consumption/Investment Model", Mathematics of Operations Research 15, 80-128.

Karatzas, I., J.P. LehoczKy, S.E. ShrevE, AND G.-L. XU (1991), "Martingale and Duality Methods for Utility Maximization in an Incomplete Market", SIAM Journal of Control and Optimization 29, 702-730.

Karatzas, I. AND S.E. SHReve (1988), Brownian Motion and Stochastic Calculus, Springer-Verlag, New York.

Lindenberg, E. (1979), "Capital Market Equilibrium with Price Affecting Institutional Investors", in: E.J. Elton and M.J. Gruber, Portfolio Theory 25 Years After, North Holland, Amsterdam.

LuenBerger, D.G. (1969), Optimization by Vector Space Methods, Wiley, New York.

MA, J., P. ProtTer, AND J. YONG (1994), "Solving Forward-Backward Stochastic Differential Equations Explicitly-A Four Step Scheme", Probability Theory and Related Fields 98, 339-359.

Pardoux, E., AND S.G. Peng (1990), "Adapted Solution of a Backward Stochastic Differential Equation", Systems \& Control Letters 14, 55-61.

Rockafellar, R.T. (1970), Convex Analysis, Princeton University Press, Princeton.

SUNDARESAN, M. (1984), "Consumption and Equilibrium Interest Rates in Stochastic Production Economies", Journal of Finance 39, 77-92.

XU, G.-L. AND S.E. Shreve (1992a), "A Duality Method for Optimal Consumption and Investment under Short-Selling Prohibition. I. General Market Coefficients", Annals of Applied Probability 2, 87-112.

XU, G.-L. AND S.E. Shreve (1992b), "A. Duality Method for Optimal Consumption and Investment under Short-Selling Prohibition. II. Constant Market Coefficients", Annals of Applied Probability 2, 314-328. 